\title{
A Fluid Model of a Traffic Network with Information Feedback and Onramp Controls
}

\author{
J. William Helton ${ }^{1} \cdot$ Frank P. Kelly ${ }^{2} \cdot$ Ruth J. Williams ${ }^{1}\left[\mathbb{D} \cdot\right.$ Ilze Ziedins $^{3}$
}

Accepted: 18 March 2021 / Published online: 18 April 2021

(C) The Author(s) 2021

\begin{abstract}
Unlimited access to a motorway network can, in overloaded conditions, cause a loss of throughput. Ramp metering, by controlling access to the motorway at onramps, can help avoid this loss of throughput. The queues that form at onramps are dependent on the metering rates chosen at the onramps, and these choices affect how the capacities of different motorway sections are shared amongst competing flows. In this paper we perform an analytical study of a fluid, or differential equation, model of a linear network topology with onramp queues. The model allows for adaptive arrivals, in the sense that the rate at which external traffic enters the queue at an onramp can depend on the current perceived delay in that queue. The model also includes a ramp metering policy which uses global onramp queue length information to determine the rate at which traffic enters the motorway from each onramp. This ramp metering policy minimizes the maximum delay over all onramps and produces equal delay times over many onramps. The paper characterizes both the dynamics and the equilibrium behavior of the system under this policy. While we consider an idealized model that leaves
\end{abstract}

This research was supported in part by National Science Foundation Grants DMS-1500835, DMS-1206772, DMS-1712974, the Charles Lee Powell Foundation, Marsden Fund grant UOA1114 and Te Pūnaha Matatini.

$凶$ Ruth J. Williams

rjwilliams@ucsd.edu

J. William Helton

helton@ucsd.edu

Frank P. Kelly

F.P.Kelly@statslab.cam.ac.uk

Ilze Ziedins

i.ziedins@auckland.ac.nz

1 Department of Mathematics, University of California, San Diego, CA 92093-0112, USA

2 Statistical Laboratory, Centre for Mathematical Sciences, University of Cambridge, Wilberforce Road, Cambridge CB3 OWB, UK

3 Department of Statistics, University of Auckland, Auckland 1142, New Zealand 
out many practical details, an aim of the paper is to develop analytical methods that yield interesting qualitative insights and might be adapted to more general contexts. The paper can be considered as a step in developing an analytical approach towards studying more complex network topologies and incorporating other model features.

Keywords Dynamic traffic network model · Ramp metering · Global delay minimization · Adaptive arrivals · Feedback signals · Equilibrium states · Long run behavior

Mathematics Subject Classification 90B10 $\cdot 90 \mathrm{~B} 20 \cdot 93 \mathrm{~B} 52 \cdot 37 \mathrm{~N} 35$

\section{Introduction}

Once motorway traffic exceeds a certain threshold level (measured in terms of density - the number of vehicles per mile) both vehicle speed and vehicle throughput drop precipitously $[8,17]$. The smooth pattern of flow that existed at lower densities breaks down, and drivers experience stop-go traffic. Maximum vehicle throughput (measured in terms of the number of vehicles per minute) occurs at quite high speedsabout 60 miles per hour on Californian freeways and on London's orbital motorway, the M25-while after flow breakdown the average speed may drop to 20-30 miles per hour. Particularly problematic is that flow breakdown may persist long after the conditions that provoked its onset have disappeared.

Ramp metering (signals on the onramps to control access to the motorway) can limit the density of vehicles, and thus can avoid the loss of throughput $[13,15,18]$. But there are many choices of metering rates that keep the density of vehicles below threshold levels, and difficult choices need to be made: if more traffic is allowed to join the motorway at one onramp then less may be allowed to join at the next onramp, with consequences for the queueing times at the different onramps.

Early work on ramp metering considered just a single ramp meter, with rates determined by historical data, and in later work, by real-time data. More recent work has concentrated on integrated network traffic control, where ramp meter rates are set in a coordinated fashion. Papageorgiou and Kotsialos [15] give a survey of earlier literature, and demonstrate, via a simulation based on historical data, the power of coordinated controls to reduce congestion. Zhang and Wang [19] give a nice survey of more recent work on coordinated ramp controls, and propose a hierarchical control approach, where the choice of ramp meter settings is given as a multiobjective optimization problem with priorities attached to the varying objectives.

Sections of the motorway are often modelled as compartments, as in the cell transmission model first proposed by Daganzo [4,5], where the emphasis is on modelling flow along the motorway, and the speed of flow within compartments and from compartment to compartment is modelled in some detail. Particularly notable here is Gomes et al. [9], which shows that in a discrete time cell transmission model with stationary demand, a linear motorway will experience a number of bottleneck points with behaviour upstream linked to their capacity, a feature we shall see arises, in the form of choke points, in the model we study below. In the setting of the cell transmission 
model in continuous time with constant demand, Coogan and Arcak [3] develop a ramp metering rule that optimizes throughput, and for a linear network, again displays the bottleneck features seen earlier in Gomes et al. Furthermore, when demand exceeds capacity, there is a unique equilibrium flow within the network.

A common assumption in these papers and other earlier work on ramp meters is that while controls and delays may affect traffic assignment and routing, the external demand remains fixed, or if not fixed, varies over time independently of the control. For an example of the latter, see Sumalee et al. [16], where Gaussian noise is added to an underlying deterministic demand, which can vary over time. In a very nice recent paper, Mandjes and Storm [14] model stochastic demand using Poisson arrival streams, and their results can be generalized to time varying arrival rates, but again, those rates do not depend on the current state of their network. However, as real time information about network delays increases in both accuracy and availability, it will be increasingly important to understand interactions between controls and demand.

This paper analyzes the effects of dynamic external demand, which decreases as delay increases, interacting with a controller obtained by minimizing the maximum delay over all onramps. The emphasis here is on the queueing delays at onramps. We make the simplifying assumption that there is a maximal carrying capacity on each section of the motorway, which cannot be exceeded, but do not include in this model the details of flow along the motorway. The maximal carrying capacity could be chosen in various ways, e.g. to be some fraction of the maximum density, or the maximal capacity under which free flow at a certain speed can be maintained.

The addition of dynamic demand raises interesting questions. With this additional feature, do the controls (and flows) converge to a unique equilibrium? And does a bottleneck phenomenon similar to that described in Gomes et al. arise? We show below that, with dynamic demand and under mild assumptions, the answer to both these questions is yes. Furthermore, we show that the dynamics of the system are governed by bottlenecks (called chokepoints here), whose locations can change dynamically with time.

\subsection{The Control Policy we Analyze}

In this paper we develop a modeling approach, and associated analytical methods, to explore control of ramp metering rates in situations where the potential motorway drivers know the current (perceived) wait time for motorway access (at each onramp). This driver behavior is incorporated in our model by allowing the rate of arrival of external traffic to an onramp (called the arrival rate function) to be a decreasing function of the current queue length there. For this situation we describe a "delay minimizing" ramp metering control policy and analyze how onramp queue lengths behave when it is in effect. Our methods are analytical, rather than simulation based. This allows us to deduce qualitative information that does not depend on details of the model such as fine structure of the arrival rate function.

The control policy we study uses knowledge of the queue lengths $m_{i}$ at each onramp $i$ and is designed to minimize the current maximum wait time (delay) over all onramps, subject to the constraint that traffic on the motorway does not exceed its capacity. We 
characterize this policy using a linear program (hence easily implementable provided one knows the $m_{i}$ ), and then analyze properties of the controlled system. The system behavior turns out to be dominated by traffic "bottlenecks" (we call them choke points). An interesting result is that under this minmax delay controller, all onramps upstream of the first bottleneck experience the same delay as the bottleneck onramp. If there is a second bottleneck, then all onramps between the first and second experience the same delay as the second bottleneck, which is less than that experienced at and upstream of the first bottleneck, and so on. So the ramp metering policy developed in this paper will yield the same delays at all onramps if there is just a single bottleneck, i.e., it is located at the final onramp in the sequence. Moreover, the approach in this paper can be modified to produce prescribed ratios of delays along the onramps.

The paper also proves (under mild assumptions) that the delay $d_{i}(t)$ at time $t$ at the $i^{t h}$ onramp converges to an equilibrium as $t \rightarrow \infty$. Bottleneck locations are functions of time and we prove that they also converge as $t \rightarrow \infty$. We prove this initially for the first bottleneck and then extend the results to downstream onramps with some additional assumptions. While not conclusive about practical performance, this gives evidence that the system is not prone to chattering (e.g. a bottleneck perpetually jumping back and forth from one onramp to another, or delays varying capriciously). We emphasize that all of these findings depend only on the monotonicity of the arrival rate function and do not depend on its detailed form.

The model can be used to explore questions such as where bottlenecks arise; what happens to throughput when capacities (in some literature these are called target flows) are changed. Thus it can be used to address issues such as where additional capacity should be added to obtain the most benefit; and where should capacity be added in order to equalize delays at all onramps. The detailed answers to these questions will depend on the particular form of the arrival rate function, however.

\subsection{Modeling Choices}

One of the assumptions in our model is that both the controller and the drivers in the arrival stream are operating with the same information on the delay at each onramp. One justification for this situation is that drivers can now access crude, lagged estimates of queueing delays from information sources such as traffic apps. Possibly, if control policies such as the one considered here were implemented, then more accurate and timely information would be widely distributed.

We deliberately do not model the measurement and estimation of vehicle volumes, an essential aspect of the closed loop control used to link ramp inflow to the target flows on the motorway in works such as [1].

In addition, much of the more detailed information practically available pertains to the state of traffic flow on the motorway and is approximated in our model by a simple constraint that flow does not exceed given capacity constraints. Refining the model much, to add realism, would likely force one to abandon analytic methods and be approachable only via simulations. In contrast to refinements, future generalizations in various directions might well be possible. 
We address equity by choosing to minimize the maximum delay over all of the onramps. Other approaches to equity have been to equalize the average delay at all onramps (see e.g. Zhang and Wang [19]); that article did not allow arrivals to adapt to the perceived delay however.

There are many different models in the literature. For example, contrasting with our work is the recent paper of Como and Nilsson [2], which is an analytical study of a model for the control of motorway outflows. On the other hand, after analysis of a traffic model, Coogan and Arcak [3] introduce a linear programming approach to ramp metering.

\subsection{Generalizations and a Guide for the Reader}

The next section states our model and results precisely. Section 3 presents key differential equations and a few very accessible facts, including properties of the equilibria of the system. Section 4 presents the (rather involved) proofs that delays and choke points have good asymptotic behavior. The final section discusses some of the subtleties of the minmax delay controller and describes more general motorway models and extensions of the minmax delay controller to them. One extension is the use of weights to adjust the wait times at onramps. Another is how to treat motorways with offramps (in addition to onramps).

\section{Model Description and Main Results}

In this section we give a detailed description of our model, and the minmax delay controller that we study in this paper. We outline the main results of the paper, including a characterization of the equilibrium behavior and dynamics of the model.

\subsection{Model Formulation}

\subsubsection{Traffic Network Model Setup}

We consider a fluid (differential equation) model for the flow of traffic in a linear network with onramp metering control and feedback of congestion information. The notation used here is similar to that of Kelly and Williams [12], where a related model was first introduced. Our model can be imagined as one for the flow of traffic on a motorway leading to a city centre, where all traffic has a common final destination. A variant of this model permits traffic to depart the network at off ramps before the final section of motorway is reached (see Sect. 5.2). (While our basic model structure is similar to that in [12], they studied a model with given (stochastic) arrivals under a proportionally fair onramp metering policy. Here we consider a model with adaptable deterministic arrivals under an onramp metering policy that minimizes the overall maximum delay. Note also that the labelling of motorway sections in this paper is in the reverse order from that in [12].) 
The linear traffic network has successive sections of motorway labelled $1,2, \ldots, N$. Traffic entering at section $i$ passes through all of the sections $j \geq i$ before leaving the network (see Fig. 1). We assume that section $i$ has a fixed carrying capacity $C_{i}$, $1 \leq i \leq N$, with $0<C_{1}<C_{2}<C_{3}<\cdots<C_{N}<\infty$. The capacity $C_{i}$ is the maximum rate at which traffic can flow along the $i t h$ section of the motorway. We assume that the only sources of traffic for the motorway come from waiting lines or queues at onramps for each of the $N$ sections of motorway. We also assume that traffic entering section $i$ at rate $\Lambda_{i}$ simultaneously uses the same capacity $\Lambda_{i}$ from each of the sections $i, i+1, \ldots, N$. This modeling assumption corresponds to a time-scale separation (refered to as the snapshot principle in [12]) where the time for onramp queues to vary is much longer than the time to pass through sections of the road. (The model can be modified to allow exogenous inflow of traffic to section 1 at a positive constant rate $C_{0}<C_{1}$. This can be analyzed in a similar manner to what we do here, by replacing $C_{i}$ by $\tilde{C}_{i}=C_{i}-C_{0}$. Consequently, we only treat the $C_{0}=0$ case here.)

Let $m_{i}(t)$ denote the size of the $i t h$ queue, at the onramp to the $i t h$ section of the motorway, at time $t$, and let $m(t)=\left(m_{1}(t), m_{2}(t), \ldots, m_{N}(t)\right)$. From a systems perspective, $m(t)$ is the state of our system at time $t$. We assume that $m$ is a Lipschitz continuous function of time that takes values in $\mathbb{R}_{+}^{N}$. In particular, $m_{i}(t)$ is non-negative and real-valued. This is consistent with a fluid model of traffic flow, in which we regard $m_{i}(t)$ as the amount (or mass) of traffic queued at onramp $i$ at time $t$, and arrivals to and departures from the queue will be flows into and flows out of the queue.

We assume that queue $i$, for $1 \leq i \leq N$, has a ramp meter which controls the rate at which traffic flows from that queue onto the $i t h$ section of the motorway. This paper examines a natural algorithm for selecting the vector of ramp meter rates as a function $\Lambda: \mathbb{R}_{+}^{N} \rightarrow \mathbb{R}_{+}^{N}$ of the current state of the system, so that $\Lambda_{i}(m(t))$ is the ramp meter rate for queue $i$ at time $t$, given the system state $m(t)$ at time $t$. This vector of rates $\Lambda(m(t))=\left(\Lambda_{1}(m(t)), \ldots, \Lambda_{N}(m(t))\right)$ needs to be chosen so that the rate of flow of traffic on any section of the motorway does not exceed its capacity, i.e.,

$$
\sum_{i: i \leq j} \Lambda_{i}(m(t)) \leq C_{j}, \quad 1 \leq j \leq N
$$

We only consider ramp meter functions $\Lambda(\cdot)$ that have the property that for each $m \in \mathbb{R}_{+}^{N}$ and $i \in\{1, \ldots, N\}, \Lambda_{i}(m)>0$ whenever $m_{i}>0$.

Given a ramp meter function $\Lambda: \mathbb{R}_{+}^{N} \rightarrow \mathbb{R}_{+}^{N}$ and $m \in \mathbb{R}_{+}^{N}$, we define for $i=$ $1, \ldots, N$,

$$
d_{i}(m):= \begin{cases}\frac{m_{i}}{\Lambda_{i}(m)} & \text { if } m_{i}>0 \\ 0 & \text { if } m_{i}=0\end{cases}
$$

We call $d_{i}(m)$ the current delay at queue $i$ associated with the state $m$. Given the current state $m(t)$ of the system, the current delay at queue $i$ is defined to be $d_{i}(m(t))$. This is the amount of time that queue $i$ would take to clear if no further traffic entered it after time $t$, and the current metering rate $\Lambda_{i}(m(t))$ were to remain constant until the queue clears. 


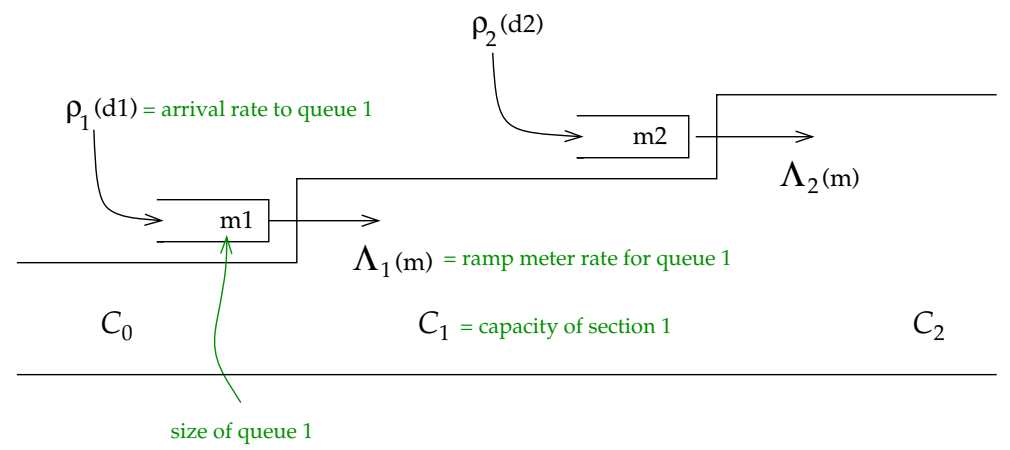

Fig. 1 Schematic of the ramp meter model with two onramps, $N=2$. Upstream is to the left and downstream is to the right of a given location. The motorway gains capacity as one moves downstream. Here $d 1=d_{1}, d 2=d_{2}, m 1=m_{1}$ and $m 2=m_{2}$

The arrival rate to queue $i$ is adaptive in the sense that it is determined by a function $\rho_{i}: \mathbb{R}_{+} \rightarrow(0, \infty)$ of the current delay at queue $i$, so that $\rho_{i}\left(d_{i}(m(t))\right)$ is the arrival rate to queue $i$ at time $t$. We assume that $\rho_{i}$ is a uniformly Lipschitz continuous and strictly decreasing function, and that $\rho_{i}(s) \rightarrow 0$ as $s \rightarrow \infty$. Let $\rho=\left(\rho_{1}, \ldots, \rho_{N}\right)$ and call it the arrival rate function for the system. We shall later impose some additional conditions on $\rho$ in order to study the long run dynamic behavior of our system (see Sect. 2.3).

\subsubsection{System Dynamics}

Given a ramp meter function $\Lambda$ and the arrival rate function $\rho$, we assume that for each $i$, the function $m_{i}:[0, \infty) \rightarrow \mathbb{R}_{+}$is Lipschitz continuous and evolves as follows (for more detail see Sect. 3.1). For almost every $t>0$,

$$
\frac{d^{+}}{d t} m_{i}(t)=\rho_{i}\left(d_{i}(m(t))\right)-\Lambda_{i}(m(t)), \quad 1 \leq i \leq N,
$$

where $\frac{d^{+}}{d t}$ is the usual right hand derivative. Of course, at a time $t>0$ where $m_{i}(t)$ is differentiable, $\frac{d^{+}}{d t}$ is the usual derivative. However, as we shall see, due to the nonnegativity constraints on $m_{i}$, and control switching from one regime to another, our $m_{i}(\cdot)$ can have points where it is not differentiable. We do not assume that there is a unique solution of the above system of differential equations, but simply that $m(\cdot)$ satisfies this system.

Remark 2.1 To ensure that $m_{i}(\cdot)$ stays in $\mathbb{R}_{+}$, we want at times $t>0$ where $m_{i}(t)=0$ that the right member of (2.3) is non-negative. We will ensure this by assuming that $\Lambda_{i}(m)=0$ when $m_{i}=0$. This ensures that if $m_{i}(\cdot)$ reaches zero, then it will immediately return to a strictly positive value, since $d_{i}(m)=0$ if $m_{i}=0$ and $\rho_{i}(0)>0$. Although this assumption means that there is no onflow to the motorway from onramp $i$ when the queue there is empty, we will show that the set of times at which this occurs is negligible and so the choice of $\Lambda_{i}(m)$ at such points is not of practical significance. 
Nevertheless, other choices may be possible if we make additional assumptions. For example, if we assume that $\rho_{i}(0)>C_{i}$, then no matter how $\Lambda_{i}(m)$ is defined when $m_{i}=0$, the right hand derivative of $m_{i}(\cdot)$ when $m_{i}(t)=0$ will be strictly positive, whenever it exists.

\subsubsection{Ramp Meter Rate Control Policy}

We shall analyze the system dynamics when the ramp meter rate function $\Lambda: \mathbb{R}_{+}^{N} \rightarrow$ $\mathbb{R}_{+}^{N}$ is chosen such that for each $m, \Lambda(m)$ minimizes the maximum delay at all onramps. More precisely, for fixed $m \in \mathbb{R}_{+}^{N}, \Lambda(m)$ is chosen to be a minimizer for the following minimization problem:

$$
\operatorname{minimize}_{\Lambda \in \mathbb{R}_{+}^{N}} \max _{1 \leq i \leq N}\left\{\frac{m_{i}}{\Lambda_{i}}\right\} \text { such that } \sum_{i: i \leq j} \Lambda_{i} \leq C_{j} \text { for } 1 \leq j \leq N
$$

In the above, we make the convention that $m_{i} / \Lambda_{i}=0$ if $m_{i}=0$ and equals $+\infty$ if $m_{i}>0, \Lambda_{i}=0$. The latter ensures that $\Lambda_{i}(m)>0$ if $m_{i}>0$ and that the value of the optimization problem is zero if $m \equiv 0$, in which case, we shall take $\Lambda(m) \equiv 0$.

Fortunately, despite the seeming nonlinearity of (2.4), upon denoting $\max _{1 \leq i \leq N} \frac{m_{i}}{\Lambda_{i}}$ as $d$ and letting $\lambda_{i}=d \Lambda_{i}$, one can see how to find such a control policy $\Lambda(\cdot)$ by solving the following linear program in the variables $d, \lambda$. (A technical point: if $m_{i}=0$, then we take $\Lambda_{i}(m)=0$, and their ratio $d_{i}(0)$ is set to be 0 (as in (2.2)), and if $m_{i} \neq 0$ the constraints force $\Lambda_{i}(m) \neq 0$. This allows us to pass back and forth between (2.4) and (2.5)-(2.7).)

Minmax delay controller $\mathbf{L P}$ For fixed $m \in \mathbb{R}_{+}^{N}$, let $\left(d_{1}^{*}(m), \lambda(m)\right)$ be a minimizing solution for the following linear program:

$$
d_{1}^{*}(m) \doteq \min _{\lambda \in \mathbb{R}_{+}^{N}} d
$$

such that

$$
\begin{aligned}
m_{i} \leq \lambda_{i}, & 1 \leq i \leq N \\
\sum_{i: i \leq j} \lambda_{i} \leq d C_{j}, & 1 \leq j \leq N
\end{aligned}
$$

When the optimal value $d_{1}^{*}(m) \neq 0$, for a minimizing solution $\left(d_{1}^{*}(m), \lambda(m)\right)$, we define the control policy $\Lambda_{i}(m)$ for $i=1,2, \ldots, N$, by

$$
\Lambda_{i}(m):= \begin{cases}\frac{\lambda_{i}(m)}{d_{1}^{*}(m)} & \text { if } m_{i}>0 \\ 0 & \text { if } m_{i}=0\end{cases}
$$

When the optimal value $d_{1}^{*}(m)=0$, then we define $\Lambda_{i}(m)=0$ for $i=1, \ldots, N$.

We warn the reader that the above LP may not determine the control policy uniquely. In particular, when the constraint (2.7) is not binding, the $\lambda_{i}(m)$ may be not uniquely 
determined. Later, when better motivated, we shall add conditions which determine the control policy uniquely (see Sect. 2.2.2).

Much of the rest of the paper is devoted to analyzing the system (2.3) when a minmax delay controller is used for it.

\subsection{Properties of the Control Policy}

The minmax delay controller LP can be solved explicitly for all onramps upstream of a certain onramp, which we call the first chokepoint (defined formally below). In the first subsection we give expressions for the optimal control policy upstream of that first chokepoint and show that delays at all these upstream onramps are equal. Downstream of the first chokepoint, the minmax delay controller LP does not give unique solutions. In the second subsection we propose a successive delay minimization algorithm for the onramps downstream of the first chokepoint, and give expressions for the optimal rate control and delays at those onramps under this algorithm.

\subsubsection{Formulas for the Controller Upstream of the First Choke Point}

The minmax delay controller LP for the linear traffic network considered here can be solved explicitly.

Given $m \in \mathbb{R}_{+}^{N}$, let $M_{j}=\sum_{i \leq j} m_{i}$ for $j=1,2, \ldots, N$. Since $\lambda_{i}=m_{i}, i=$ $1,2, \ldots, N$ is feasible for the linear program, it is easy to see that the value of the LP is

$$
d_{1}^{*}(m):=\max _{j} \frac{M_{j}}{C_{j}} .
$$

Let $j_{1}(m)=\max \left\{j: M_{j} / C_{j}=d_{1}^{*}(m)\right\}$, and call it the (first) choke point associated with $m$. Let $J_{1}(m)=\left\{j: j \leq j_{1}(m)\right\}$, the set containing $j_{1}(m)$ and onramps upstream from $j_{1}(m)$, and $\tilde{J}_{1}(m)=\left\{j: j>j_{1}(m)\right\}$, the complement of $J_{1}(m)$, containing onramps downstream from $j_{1}(m)$. It is straightforward to see from the above that $d_{1}^{*}(\cdot)$ is a continuous function on $\mathbb{R}_{+}^{N}$ and $j_{1}(\cdot)$ is an upper semicontinuous function on $\mathbb{R}_{+}^{N}$, i.e., $\lim \sup _{m \rightarrow m_{0}} j_{1}(m) \leq j_{1}\left(m_{0}\right)$, for all $m_{0} \in \mathbb{R}_{+}^{N}$.

Note that if $m=0$, then $d_{1}^{*}(m)=0$ and recall that if $m_{i}=0$, then $d_{i}(m)=0$. Henceforth, the notation $m>0$ will mean that $m_{i}>0$ for $i=1, \ldots, N$. The next proposition characterizes the behavior of the delays at, or upstream of, the first chokepoint when $m>0$.

Proposition 2.2 Fix $m \in \mathbb{R}_{+}^{n}$ satisfying $m>0$. Then

1. $d_{i}(m)=d_{1}^{*}(m)$, for $i=1, \ldots, j_{1}(m)$.

2. $\lambda_{i}(m)=m_{i}, \quad \Lambda_{i}(m)=\frac{m_{i}}{d_{1}^{*}(m)} \quad$ for $i=1, \ldots, j_{1}(m)$.

3. $\sum_{i \leq j_{1}(m)} \Lambda_{i}(m)=C_{j_{1}(m)}$.

4. The solutions $\lambda_{i}(m): 1 \leq i \leq j_{1}(m)$ and $d_{1}^{*}(m)$ to the LP are unique. 
The proposition tells us that on $\left\{m \in \mathbb{R}_{+}^{N}: m>0\right\}$, the control values $\Lambda_{i}(\cdot)$ for $i \leq j_{1}(m)$, i.e., at or upstream from the first choke point, have excellent properties. Downstream values are highly nonunique and Sect. 2.2.2 addresses that.

Proof Fix $m>0$. Then $d_{1}^{*}(m)>0$ and a minimizing solution, $\lambda(m)$, for the LP satisfies

$$
M_{j_{1}(m)}=\sum_{i \leq j_{1}(m)} m_{i} \leq \sum_{i \leq j_{1}(m)} \lambda_{i}(m) \leq d_{1}^{*}(m) C_{j_{1}(m)}=\frac{M_{j_{1}(m)}}{C_{j_{1}(m)}} C_{j_{1}(m)}=M_{j_{1}(m)},
$$

and so all of the inequalities are equalities. Since $m_{i} \leq \lambda_{i}(m)$ for all $i$, we then obtain $m_{i}=\lambda_{i}(m)$ for $i \leq j_{1}(m)$, and by the definition of $\Lambda(m)$,

$$
d_{1}^{*}(m) \Lambda_{i}(m)=\lambda_{i}(m)=m_{i} \text { for } i=1, \ldots, j_{1}(m) .
$$

So for $i \leq j_{1}(m), \Lambda_{i}(m)=m_{i} / d_{1}^{*}(m)$ is uniquely determined, and the delay $d_{i}(m)$ at ramp $i$ is $\frac{m_{i}}{\Lambda_{i}(m)}=d_{1}^{*}(m)$. This establishes Items 1 and 2 of the proposition.

Furthermore, by (2.10),

$$
\sum_{i \leq j_{1}(m)} \Lambda_{i}(m)=\frac{\sum_{i \leq j_{1}(m)} \lambda_{i}(m)}{d_{1}^{*}(m)}=C_{j_{1}(m)}
$$

and Item 3 follows.

The uniqueness in Item 4 follows immediately from the formulas in (2.9) and Item 2.

\subsubsection{Successive Delay Minimization Controller}

For $m \in \mathbb{R}_{+}^{N}$, although the $\Lambda_{i}(m)$ are uniquely determined at and upstream of the first choke point, that is for $i \leq j_{1}(m)$, this is not the case for $i>j_{1}(m)$. One reasonable way to select the $\Lambda_{i}(m)$ for $i>j_{1}(m)$ is by successive maximum delay minimization, which we now describe. The basic idea is to pretend that the motorway starts at the first choke point, $j_{1}(m)$, focus on what is happening downstream from it, and just apply the minmax delay contol algorithm there. This produces its own first choke point $j_{2}(\mathrm{~m})$, which of course is the second choke point for the motorway as a whole. We proceed down the motorway in this fashion, thereby producing choke points $j_{1}(m), \ldots, j_{K(m)}(m)$ and associated successive minmax delays $d_{1}^{*}(m), \ldots, d_{K(m)}^{*}(m)$ where $K(m)$ is the total number of choke points, which depends on $m$. In a similar manner to that for the first choke point, this leads to explicit formulas for the successive minmax delays, choke points and control laws, which we now describe via an inductive procedure.

Let $d_{1}^{*}(m), j_{1}(m), J_{1}(m)$ be defined as in Sect. 2.2.1. Suppose that successive minmax delays, $d_{1}^{*}(m), \ldots, d_{n-1}^{*}(m)$ and choke points, $1 \leq j_{1}(m)<\cdots<j_{n-1}(m)<$ 
$N$, have been defined for some $n \geq 2$. Then define

$$
\begin{aligned}
& d_{n}^{*}(m)=\max _{j>j_{n-1}(m)} \frac{M_{j}-M_{j_{n-1}(m)}}{C_{j}-C_{j_{n-1}(m)}}, \\
& j_{n}(m)=\max \left\{j>j_{n-1}(m): d_{n}^{*}(m)=\frac{M_{j}-M_{j_{n-1}(m)}}{C_{j}-C_{j_{n-1}(m)}}\right\}, \\
& J_{n}(m)=\left\{j: j_{n-1}(m)<j \leq j_{n}(m)\right\} .
\end{aligned}
$$

We continue in this manner until $j_{n}(m)=N$. The value of $n$ at which the latter occurs is the number of chokepoints $K(m)$ associated with $m$. For example, setting $M_{0}(m)=0, C_{0}(m)=0$, and $j_{0}(m)=0$, we readily see that the above formulas reduce to our previous definitions:

$d_{1}^{*}(m)=\max _{j} \frac{M_{j}}{C_{j}}, \quad j_{1}(m)=\max \left\{j: M_{j} / C_{j}=d_{1}^{*}(m)\right\}, \quad J_{1}(m)=\left\{j: j \leq j_{1}(m)\right\}$.

As we shall see in Lemma 3.7, for $m \in \mathbb{R}_{+}^{N}$, the $d_{i}^{*}(m)$ are decreasing in $i$.

Via this successive delay minimization procedure, a unique choice for $\lambda(m), \Lambda(m)$ is determined for $n=1, \ldots, K(m)$, and $j_{n-1}(m)<i \leq j_{n}(m)$, by

$$
\begin{aligned}
\lambda_{i}(m) & := \begin{cases}\frac{d_{1}^{*}(m) m_{i}}{d_{n}^{*}(m)} & \text { if } m_{i}>0, \\
0 & \text { if } m_{i}=0,\end{cases} \\
\Lambda_{i}(m) & := \begin{cases}\frac{m_{i}}{d_{n}^{*}(m)} & \text { if } m_{i}>0, \\
0 & \text { if } m_{i}=0 .\end{cases}
\end{aligned}
$$

We note that $\lambda(m)$ is an optimal solution of the minmax delay controller LP and $\Lambda_{i}(m)$ is the corresponding optimal control. Also, the following proposition can be proved in a similar manner to Proposition 2.2.

Proposition 2.3 Suppose the successive minmax delay control policy is in effect. Fix $m \in \mathbb{R}_{+}^{n}$ satisfying $m>0$. Then for $n=1, \ldots, K(m)$, for each $i$ such that $j_{n-1}(m)<$ $i \leq j_{n}(m)$, we have

1. $d_{i}(m)=d_{n}^{*}(m)$,

2. $\lambda_{i}(m)=\frac{d_{1}^{*}(m) m_{i}}{d_{n}^{*}(m)}, \quad \Lambda_{i}(m)=\frac{m_{i}}{d_{n}^{*}(m)}$,

3. $\sum_{j_{n-1}(m)<i \leq j_{n}(m)} \Lambda_{i}(m)=C_{j_{n}(m)}-C_{j_{n-1}(m)}$.

Proof This proposition is proved in a similar manner to Proposition 2.2, with $d_{n}^{*}(m)$, $\left\{d_{n}^{*}(m) \Lambda_{i}(m)=\tilde{\lambda}_{i}(m)=m_{i}: j_{n-1}(m)<i \leq j_{n}(m)\right\}, M_{j_{n}(m)}-M_{j_{n-1}(m)}, C_{j_{n}(m)}-$ $C_{j_{n-1}(m)}$ in place of $d_{1}^{*}(m),\left\{d_{1}^{*}(m) \Lambda_{i}(m)=\lambda_{i}(m)=m_{i}: i \leq j_{1}(m)\right\}, M_{j_{1}(m)}$, $C_{j_{1}(m)}$, respectively, and using (2.8) to obtain the formula for $\lambda_{i}(m): j_{n-1}(m)<i<$ $j_{n}(m)$.

Notational Convention From hereon, when considering solutions $\{m(t), t \geq 0\}$ of the system dynamics, we shall slightly abuse notation and abbreviate $K(m(t)), d_{n}(m(t))$ 
and $j_{n}(m(t))$ for $1 \leq n \leq K(m(t))$, and $\Lambda_{i}(m(t))$ for $1 \leq i \leq N$, as $K(t), d_{n}(t)$, and $j_{n}(t)$ for $1 \leq n \leq K(t)$, and $\Lambda_{i}(t)$ for $1 \leq i \leq N$, respectively. We also let $M_{j}(t)$ stand for $\sum_{i=1}^{j} m_{i}(t)$.

\subsection{Main Dynamic Results}

To this point we have given (an easily implemented) control law and shown that it has choke points and equal ramp delays between choke points. Now, under a mild additional condition, we study the dynamics and give evidence that it is well behaved, mitigating the worry that delays and choke points will be perpetually changing in steady state conditions. The additional mild condition is on the arrival rate functions and is specified as follows. For $j=1, \ldots, N$, define

$$
\psi_{j}(s) \doteq \frac{\sum_{i=1}^{j} \rho_{i}(s)}{C_{j}}, \quad s \geq 0 .
$$

We henceforth assume that

$$
\psi_{j}(0)>1 \text { for } j=1, \ldots, N
$$

It follows (see Lemma 3.6) that for each $j=1, \ldots, N$, there is a unique value $e_{j}$ such that

$$
\psi_{j}\left(e_{j}\right)=1
$$

Throughout the paper we shall assume that the $e_{j}$ are distinct. It turns out that $e=$ $\left(e_{1}, \ldots, e_{N}\right)$ is an important quantity in the dynamic behavior of our algorithm.

We call $e_{1}, \ldots, e_{N}$ the equilibrium delays because $e_{k}$ is the delay, which if experienced at each ramp $i=1, \ldots, k$, makes the total of all traffic coming into these ramps equal to the capacity of the motorway at onramp $k$. We also define the maximum equilibrium delay, $e^{*}$, and the last onramp at which that maximum is attained, $j^{*}$, as

$$
e^{*} \doteq \max _{i} e_{i}, \quad j^{*} \doteq \max \left\{j: e_{j}=e^{*}\right\}
$$

\subsubsection{Behavior Upstream of the First Choke Point}

We now investigate the dynamics upstream of the first choke point in greater detail.

Theorem 2.4 Assume (2.18) holds and the equilibrium delays $e_{i}, i=1, \ldots, N$ are mutually distinct. Then the motorway under the minmax delay control policy, for any initial $m(0) \in \mathbb{R}_{+}^{N}$, satisfies

1. $d_{1}^{*}(t) \rightarrow e^{*}$ as $t \rightarrow \infty$.

2. There is $t_{0} \geq 0$, depending on $m(0)$, such that for all $t \geq t_{0}$, the first choke point $j_{1}(t)$ equals $j^{*}$. 
3. If, in addition, $d_{1}^{*}\left(t_{0}\right)=e^{*}$, then $d_{1}^{*}(t)=e^{*}$ for $t \geq t_{0}$.

Items 1 and 2 provide evidence that delays and choke points usually do not jump around a lot as time goes by.

Proof Items 1 and 2 are proved in Sect. 4.2. The proof requires analysing several cases as key functions transiently switch back and forth from being active to inactive and vice versa. Item 3 follows immediately from Item 4 of Lemma 3.5 with $a=t_{0}$.

\subsubsection{Multiple Choke Points}

To analyze the successive delay minmax control policy, we think of sections of roadway along which the onramps have constant delays. The first section is the one we just analyzed. The notions surrounding equilibria for downstream motorway sections are defined as follows.

Define the cumulative sums

$$
R_{i, j}(s)=\sum_{k=i}^{j} \rho_{k}(s), \quad \text { for } s \geq 0,1 \leq i<j \leq N
$$

Let $e_{j}^{(1)}$ be the solution of $R_{1, j}(s)=C_{j}$, for $j=1,2, \ldots, N$. Let

$$
e_{1}^{*}=\max _{j} e_{j}^{(1)} \quad j_{1}^{*}=\max \left\{j: e_{j}^{(1)}=e_{1}^{*}\right\},
$$

which yield our previous definitions via $e_{j}^{(1)}=e_{j}$ and $e_{1}^{*}=e^{*}, j_{1}^{*}=j^{*}$ as defined above. Also we set $j_{0}=j_{0}^{*}=0$, and recall that $C_{0}=0$.

To analyze downstream sections of motorway we make the following inductive definition. Suppose $e_{k}^{*}$ and $j_{k}^{*}$ exist, and that $j_{k}^{*}<N$, for some $k \in\{1,2, \ldots, N-1\}$. Then, assuming (2.18), let $e_{j}^{(k+1)}$ be the solution of

$$
R_{j_{k}^{*}+1, j}(s)=C_{j}-C_{j_{k}^{*}}, \quad s \geq 0,
$$

for $j=j_{k}^{*}+1, j_{k}^{*}+2, \ldots, N$. Let

$$
e_{k+1}^{*}=\max _{j>j_{k}^{*}} e_{j}^{(k+1)} \quad j_{k+1}^{*}=\max \left\{j>j_{k}^{*}: e_{j}^{(k+1)}=e_{k+1}^{*}\right\} .
$$

Note that since $N$ is finite, there exists a $k \leq N$ such that $j_{k}^{*}=N$, which we denote by $K$, i.e., $K$ is the number of equilibrium choke points.

Theorem 2.5 Assume that for each $k=1, \ldots, K$, the equilibria $e_{j}^{(k)}, j=j_{k-1}^{*}+1$, $\ldots, N$, are distinct. Suppose for some $k \in\{1,2, \ldots, K-1\}$, that there is a finite time $t_{k}$ (depending on $m(0)$ ) such that the chokepoints up through $k$ satisfy

$$
j_{n}(t)=j_{n}^{*} \text { and } d_{n}^{*}(t)=e_{n}^{*} \text { for all } t \geq t_{k}, 1 \leq n \leq k
$$


Then

1. $d_{k+1}^{*}(t) \rightarrow e_{k+1}^{*}$ as $t \rightarrow \infty$,

2. there exists a finite $t_{k+1} \geq t_{k}$ such that $j_{k+1}(t)=j_{k+1}^{*}$ for $t \geq t_{k+1}$.

Proof The hypothesis guarantees that $j_{1}^{*}, j_{2}^{*}, \ldots, j_{k}^{*}$ remain chokepoints after time $t_{k}$. So, summing in Item 3 of Proposition 2.3, ramp meter rates at each chokepoint up to the $k^{\text {th }}$ satisfy

$$
\sum_{i: i \leq j_{n}^{*}} \Lambda_{i}(m(t))=C_{j_{n}^{*}}, \quad 1 \leq n \leq k, \quad t \geq t_{k} .
$$

In other words, all capacity at each chokepoint up to the $k^{\text {th }}$ is in use. Furthermore, for $t \geq t_{k}$

$$
\begin{aligned}
M_{j_{k}^{*}}(t)=\sum_{i: i \leq j_{k}^{*}} m_{i}(t) & =\sum_{i: i \leq j_{k}^{*}} d_{i}(t) \Lambda_{i}(m(t)) \\
& =\sum_{i: i \leq j_{1}^{*}} d_{i}(t) \Lambda_{i}(m(t))+\sum_{n=2}^{k} \sum_{i=j_{n-1}^{*}+1}^{j_{n}^{*}} d_{i}(t) \Lambda_{i}(m(t)) \\
& =e_{1}^{*} C_{j_{1}^{*}}+\sum_{n=2}^{k} e_{n}^{*}\left(C_{j_{n}^{*}}-C_{j_{n-1}^{*}}\right)
\end{aligned}
$$

the final equality following since we have assumed that the delay, $d_{i}(t)$ at each onramp in the $n^{\text {th }}$ subsection for $1 \leq n \leq k$ is given by $e_{n}^{*}$, and also, from (2.22) the ramp meter rates between the $(n-1)^{s t}$ and $n^{t h}$ chokepoints satisfy $\sum_{i=j_{n-1}^{*}+1}^{j_{n}^{*}} \Lambda_{i}(m(t))=$ $C_{j_{n}^{*}}-C_{j_{n-1}^{*}}$.

Since the total onflow, $\sum_{i: i \leq j_{k}^{*}} \Lambda_{i}(m(t))$, up to $j_{k}^{*}$ is constant, and $M_{j_{k}^{*}}(t)$ is constant for $t \geq t_{k}$, the motorway sections having onramps $j_{k}^{*}+1, \ldots, N$ behave as sections of motorway treated in Sect. 2.3.1, but with carrying capacity $C_{j}-C_{j_{k}^{*}}$ at onramp $j$, and cumulative queue lengths $M_{j}(t)$ up to onramp $j$ replaced by $M_{j}(t)-\left[e_{1}^{*} C_{j_{1}^{*}}+\right.$ $\left.\sum_{n=2}^{k} e_{n}^{*}\left(C_{j_{n}^{*}}-C_{j_{n-1}^{*}}\right)\right]$, for $j_{k}^{*}<j \leq N$. The result then follows from an application of Theorem 2.4 to the reduced motorway model associated with onramps downstream of onramp $j_{k}^{*}$.

This theorem provides support for the following more general conjecture. To prove the conjecture would require involved estimates showing that if upstream of chokepoint $j_{k}$ the delays are near enough to equilibrium, then the conclusion of Theorem 2.5 still holds. Such a proof would be very long and involved and we have not attempted it here.

Conjecture 2.6 For each $k: 1 \leq k \leq K$ and initial conditions $m(0)$, there exists a $t_{k}$, dependent on $m(0)$, such that for $t \geq t_{k}$, the delay time $d_{k}^{*}(t)$ exists, with choke point $j_{k}(t) \leq N$, and, furthermore, 
1. $d_{k}^{*}(t) \rightarrow e_{k}^{*}$ as $t \rightarrow \infty$,

2. $j_{k}(t) \rightarrow j_{k}^{*}$ as $t \rightarrow \infty$.

\subsection{Other Control Perspectives}

In control theory language, the problem studied here is: Consider a system that satisfies the following differential equation for almost all $t>0$.

$$
\frac{d}{d t} m(t)=\Xi(m(t), \Lambda(m(t)))
$$

where

$$
\Xi_{i}(m, \Lambda) \doteq \begin{cases}\rho_{i}\left(\frac{m_{i}}{\Lambda_{i}(m)}\right)-\Lambda_{i}(m) & \text { if } m_{i}>0 \\ \rho_{i}(0) & \text { if } m_{i}=0\end{cases}
$$

The state of the system is $m$ and we seek a state feedback law $\Lambda(m)$ which minimizes the cost $d(m) \doteq \max _{i} \frac{m_{i}}{\Lambda_{i}(m)}$.

There is a classical theory which pertains to problems of this type and a guide to it can be found in $[10,11]$. To such a problem we can associate a Bellman type inequality which couples the controller $u(m)$ and a "value function" $V(m)$. We pursued this but were unable (and felt it unlikely) to find a solution. Even had we found a solution, proving asymptotic stability of the controlled system seems not to result immediately from that theory.

\section{Key Differential Equations and Basic Properties}

\subsection{Basics of Queue Length Dynamics}

Recall the dynamics of the queue lengths described in Sect. 2.1.2. In particular, we assume that each of the components of the $N$-dimensional fluid queue size function, $m(\cdot)=\{m(t), t \geq 0\}$, is a Lipschitz continuous function. By Rademacher's theorem (see Sect. 3.1.6 in [7]), a Lipschitz continuous function has a finite derivative from the left and from the right at almost every (a.e.) $t>0$, these derivatives are equal a.e., and the function is absolutely continuous and can be recovered by integrating its a.e. defined (left/right) derivative.

In defining our system dynamics in Sect. 2.1.2, we assumed that for $i=1, \ldots, N$, at each of the almost every $t>0$ where the derivative from the right, $\frac{d^{+}}{d t} m_{i}(t)$, exists for $m_{i}(\cdot)$, it satisfies (2.3). Upon breaking into the cases when $m_{i}(t)>0$ and $m_{i}(t)=0$, under our conventions that $\Lambda_{i}(m)=0$ and $d_{i}(m)=0$ when $m_{i}=0$, the differential equation (2.3) for $m_{i}(\cdot)$ at time $t$ reads

$$
\frac{d^{+}}{d t} m_{i}(t)= \begin{cases}\rho_{i}\left(\frac{m_{i}(t)}{\Lambda_{i}(m(t))}\right)-\Lambda_{i}(m(t)) & \text { if } m_{i}(t)>0 \\ \rho_{i}(0) & \text { if } m_{i}(t)=0\end{cases}
$$


We shall find it convenient to define the notion of a regular point for $m(\cdot)$.

Definition 3.1 A time $t>0$ is a regular point for $m(\cdot)$, if $m_{i}(\cdot)$ is differentiable at $t$ for each $i=1, \ldots, N$. We denote the derivative of $m_{i}(\cdot)$ at a regular point for $m(\cdot)$ by $\dot{m}_{i}(t)$, or by $\frac{d}{d t} m_{i}(t)$, for $i=1, \ldots, N$.

Because $m(\cdot)$ is Lipschitz continuous, a.e. $t>0$ is a regular point for $m(\cdot)$ and the behavior of $m(\cdot)$ can be recovered from knowing its differential behavior just at its regular points. The following lemma simplifies the analysis of such points by excluding those times where some component of $m(\cdot)$ is zero.

Lemma 3.2 Suppose that $t>0$ is a regular point for $m(\cdot)$. Then for each $i=1, \ldots, N$, $m_{i}(t)>0$.

Proof For a proof by contradiction, suppose that $t>0$ is a regular point for $m(\cdot)$ and that $m_{i}(t)=0$ for some $i \in\{1, \ldots, N\}$. Then, since $m_{i}(\cdot)$ only takes non-negative values, the derivative from the left and from the right at time $t$ will be non-positive and non-negative, respectively, and so the derivative of $m_{i}(\cdot)$ at $t$ must be zero. However, from (3.1), the derivative from the right of $m_{i}(\cdot)$ at $t$ is $\rho_{i}(0)$, which is strictly positive, by assumption. This yields the desired contradiction.

Remark 3.3 To determine dynamic behavior derived from (3.1), it suffices to consider differential behavior at regular points for $m(\cdot)$. By the above lemma, we see that we can assume that $m_{i}(t)>0$ at such points and so only the upper case in equation (3.1) needs to be considered.

\subsection{Virtual Delays}

Our proofs of the main theorems heavily use the notion of virtual delays. These are functions of time defined by

$$
\delta_{i}(t)=\frac{M_{i}(t)}{C_{i}}, \quad i=1, \ldots, N,
$$

where we recall that $M_{i}(t)=\sum_{\ell \leq i} m_{\ell}(t)$. We call $\delta_{i}$ the virtual delay at queue $i$. We use the term "virtual delay" because $\delta_{i}(t)$ would be the delay at onramp $i$ if the queues at the onramps indexed by $\ell=1, \ldots, i$ were collapsed to a single queue at onramp $i$, and the ramp meter rate at onramp $i$ was equal to the maximum possible, $C_{i}$. These virtual delays are connected to the minmax controlled system through the fact that, if the first choke point is $i$, then $\delta_{i}(t)$ is the delay at queue $i$ and equals the minmax delay $d_{1}^{*}(t)$. Indeed, we can compare the size of the $\delta_{i}^{\prime} s$ to determine the minmax delay $d_{1}^{*}(t)$ and also to determine the optimal control law $\Lambda_{j}(m(t))$ for $j \leq j_{1}(t)$ (see Lemma 3.5 ).

Our analysis of the virtual delays will depend heavily on a well behaved differential equation which they satisfy. Fortunately, for each $j=1, \ldots, N$, the function $\delta_{j}(\cdot)$, being a finite sum of Lipschitz functions is Lipschitz continuous and any regular point for $m(\cdot)$ is a regular point for $\delta_{j}(\cdot)$. It follows from (3.6) below that $d_{1}^{*}(\cdot)$ is Lipschitz continuous, being a maximum of a finite number of Lipschitz continuous functions. 
Lemma 3.4 At any regular point $t>0$ for $m(\cdot)$, for $j=1,2, \ldots, N$, we have

$$
\frac{d}{d t} \delta_{j}(t)=\frac{1}{C_{j}} \sum_{i \leq j}\left(\rho_{i}\left(\frac{m_{i}(t)}{\Lambda_{i}(m(t))}\right)-\Lambda_{i}(m(t))\right) .
$$

Proof By Lemma 3.2, at any regular point $t>0$ for $m(\cdot)$, we have for each $i$ that $m_{i}(t)>0$ and so by the definition of $\Lambda(m(t))$ we have $\Lambda_{i}(m(t))>0$, thus legitimizing the right hand side of the differential equation. Now we just sum the differential equations (3.1) on $i$.

Recall, from Sect. 2.3, that for each $j \in\{1, \ldots, N\}$,

$$
\psi_{j}(s) \doteq \frac{\sum_{i=1}^{j} \rho_{i}(s)}{C_{j}}=\frac{R_{1, j}(s)}{C_{j}} \quad \text { for } s \in \mathbb{R}_{+} .
$$

The function $\rho_{i}$ is uniformly Lipschitz continuous, decreasing and bounded below, and so it is bounded on $\mathbb{R}_{+}$, for $i=1, \ldots, j$. It follows that $\psi_{j}$ is uniformly Lipschitz continuous, decreasing and bounded on $\mathbb{R}_{+}$. We define the $j^{\text {th }}$ auxiliary differential equation for $t \geq 0$ by

$$
\frac{d}{d t} u_{j}(t)=\psi_{j}\left(u_{j}(t)\right)-1
$$

It follows from standard differential equation theory, using the regularity properties of $\psi_{j}$ and the assumption (2.18), that given $u_{j}(0) \in \mathbb{R}_{+}$, there is a unique continuously differentiable solution $u_{j}:[0, \infty) \rightarrow \mathbb{R}_{+}$of (3.5) with this initial value. The equilibrium delay, $e_{j}$, introduced in Sect. 2.3, is the unique fixed point of this equation (see Lemma 3.6). Recall the definitions of $e^{*} \doteq \max _{i} e_{i}$ and $j^{*} \doteq \max \left\{j: e_{j}=e^{*}\right\}$ from (2.20).

Items $1-4$ of the following lemma indicate properties related to the differential equations (3.3) and (3.5) that play an important role in determining the asymptotic behavior of $d_{1}^{*}(t)$ as $t \rightarrow \infty$. In particular, Item 3 indicates that when $j_{1}(\cdot)=j^{\dagger}$ is constant over an interval of time, then $\delta_{j^{\dagger}}(\cdot)=d_{1}^{*}(\cdot)$ is a solution of the auxiliary equation (3.5) over the interval, with $j=j^{\dagger}$ there.

Lemma 3.5 The following properties hold when the minmax control law is in force. For each $t \geq 0$,

$$
\begin{aligned}
d_{1}^{*}(t) & =\max _{i \leq N} \delta_{i}(t) \\
\Lambda_{j}(m(t)) & =\frac{m_{j}(t)}{d_{1}^{*}(t)}=\frac{m_{j}(t)}{\delta_{j_{1}(t)}(t)}=\frac{m_{j}(t)}{M_{j_{1}(t)}(t)} C_{j_{1}(t)} \quad \text { for } j \leq j_{1}(t),
\end{aligned}
$$

where the first choke point at time $t$ is

$$
j_{1}(t)=\max \left\{j: \delta_{j}(t)=d_{1}^{*}(t)\right\}
$$


Furthermore, if $j^{\dagger} \in\{1, \ldots, N\}$ and $0 \leq a<b<\infty$ are such that the first choke point $j_{1}(t)=j^{\dagger}$ for all $t \in[a, b)$, then we have

1. for each regular point $t>0$ that is in $[a, b)$,

$$
\frac{d}{d t} \delta_{j}(t)=\psi_{j}\left(d_{1}^{*}(t)\right)-\frac{\delta_{j}(t)}{d_{1}^{*}(t)} \quad j=1,2, \ldots, j^{\dagger},
$$

2. for $j \leq j_{1}(t)=j^{\dagger}, \Lambda_{j}(m(t))=\frac{m_{j}(t)}{M_{j_{1}(t)}(t)} C_{j_{1}(t)}$ for all $t \in[a, b)$,

3. for all $t \in(a, b), d_{1}^{*}(t)=\delta_{j^{\dagger}}(t)$ satisfies the auxiliary differential equation (3.5), with $j=j^{\dagger}$ and $d_{1}^{*}$ in place of $u_{j}$,

4. if $j^{\dagger}=j^{*}$ and $d_{1}^{*}(a)=e^{*}$, then $d_{1}^{*}(t)=e^{*}$ for all $t \in[a, b)$.

Proof Equations (3.6), (3.7) and (3.8) follow immediately from (3.2), (2.9), Item 2 of Proposition 2.2, and the definition of the first choke point. Item 2 is immediate from (3.7).

For the proof of Items $1-4$, assume that $j^{\dagger}$ and $a, b$ are as in the condition immediately preceding these items. For Item 1, by Items 1 and 2 of Proposition 2.2, for all $i \leq j^{\dagger}$, we have $d_{1}^{*}(t)=d_{i}(t)=m_{i}(t) / \Lambda_{i}(m(t))$. When combined with Lemma 3.4 and the definition of $\delta_{j}$, this yields for each regular point $t>0$ that is in $[a, b)$, for $j=1, \ldots, j^{\dagger}$,

$$
\begin{aligned}
\frac{d}{d t} \delta_{j}(t) & =\frac{1}{C_{j}}\left(\sum_{i \leq j} \rho_{i}\left(d_{1}^{*}(t)\right)-\sum_{i \leq j} \frac{m_{i}(t)}{d_{1}^{*}(t)}\right) \\
& =\psi_{j}\left(d_{1}^{*}(t)\right)-\frac{\delta_{j}(t)}{d_{1}^{*}(t)} .
\end{aligned}
$$

For Item 3, note that since $d_{1}^{*}(t)=\delta_{j_{1}(t)}=\delta_{j^{\dagger}}(t)$ for all $t \in[a, b)$, Item 1 implies that $\delta_{j^{\dagger}}$ satisfies (3.5) (with $u_{j}=\delta_{j^{\dagger}}$ and $j=j^{\dagger}$ ) at all regular points $t \in(a, b)$. So by integration, $\delta_{j^{\dagger}}(t)=\delta_{j^{\dagger}}(a)+\int_{a}^{t}\left(\psi_{j^{\dagger}}\left(d_{1}^{*}(s)\right)-1\right) d s$ for all $t \in[a, b)$, and since the integrand equals the continuous function $\psi_{j^{\dagger}}\left(\delta_{j^{\dagger}}(\cdot)\right)-1$, it follows that $\delta_{j^{\dagger}}$ satisfies (3.5) at all $t \in(a, b)$.

For Item 4, suppose that $j^{\dagger}=j^{*}$ and $d_{1}^{*}(a)=e^{*}$. Then, combining Item 3 and the uniqueness of solutions of (3.5) for $j=j^{*}$ with the fact that $\psi_{j^{*}}\left(e^{*}\right)=1$, we conclude that $d_{1}^{*}(t)=\delta_{j^{*}}(t)=e^{*}$ for all $t \in[a, b)$.

\subsection{Delays $d_{k}^{*}(t)$ of the Multichoked Minmax System and Their Equilibria $e_{k}^{*}$}

\subsubsection{Properties of Equilibrium Delays $e_{1}, e_{2}, \ldots, e_{N}$}

Note that the equilibrium delays $e_{j}$, defined in Sect. 2.3, satisfy $\psi_{j}\left(e_{j}\right)=1$, for $j=1,2, \ldots, N$. In other words they are equilibria for the auxiliary differential equations in (3.5). In order to guarantee the existence of $e_{1}, e_{2}, \ldots, e_{N}$, we have 
assumed that $\psi_{j}(0)>1$ for all $j$, and a sufficient condition for this to be the case is that $\rho_{j}(0)>C_{j}$ for all $j$. The condition on the $\psi_{j}$ just says there are no parts of the motorway which are underloaded when all queues are empty. Recall that in addition we have assumed that each $\rho_{j}$ is a strictly decreasing uniformly Lipschitz continuous function that decreases to 0 at $\infty$.

Lemma 3.6 For each $j$, equation (3.5) has a unique equilibrium point $e_{j}$ and it is globally asymptotically stable, in the sense that all solutions of the equation converge to this equilibrium as time goes to infinity.

Proof Uniqueness of the equilibrium $e_{j}$ follows from strict monotonicity of $\psi_{j}$, which it inherits from the $\rho_{i}^{\prime}$ s. Existence follows from the intermediate value theorem. Global asymptotic stability comes from the facts that

$$
\psi_{j}(s)-1<0 \text { if } e_{j}<s \quad \text { and } \quad \psi_{j}(s)-1>0 \text { if } e_{j}>s .
$$

\subsubsection{The Successive $e_{k}^{*}$ and $d_{k}^{*}$ are Decreasing with $k$}

We show first that the delays $d_{k}^{*}(m)$ are strictly decreasing in $k$, and then prove that the $e_{k}^{*}$ as defined in Sect. 2.3.2 are strictly decreasing in $k$.

Lemma 3.7 Consider the minmax controlled system and recall that $K(m)$ denotes the number of chokepoints $j_{1}(m), j_{2}(m), \ldots, j_{K(m)}(m)=N$ for state $m$. Then

$$
d_{1}^{*}(m)>d_{2}^{*}(m)>\cdots>d_{K(m)}^{*}(m)
$$

Proof Fix $m \in \mathbb{R}_{+}^{N}$ and $k \in\{1,2, \ldots, K(m)-1\}$. In the following proof, we suppress the dependence of $j_{k-1}, j_{k}$ on $m$, to ease the notation. As usual, $M_{j}=\sum_{i=1}^{j} m_{i}$. By definition,

$$
d_{k}^{*}(m)=\max _{j>j_{k-1}} \frac{M_{j}-M_{j_{k-1}}}{C_{j}-C_{j_{k-1}}}=\frac{M_{j_{k}}-M_{j_{k-1}}}{C_{j_{k}}-C_{j_{k-1}}}
$$

and for all $j>j_{k}$ we have

$$
\frac{M_{j}-M_{j_{k-1}}}{C_{j}-C_{j_{k-1}}}<d_{k}^{*}(m) .
$$

This last inequality is equivalent to

$$
\begin{aligned}
& \frac{M_{j}-M_{j_{k}}+M_{j_{k}}-M_{j_{k-1}}}{C_{j}-C_{j_{k}}+C_{j_{k}}-C_{j_{k-1}}}<\frac{M_{j_{k}}-M_{j_{k-1}}}{C_{j_{k}}-C_{j_{k-1}}} \\
& \quad \Leftrightarrow\left(M_{j}-M_{j_{k}}+M_{j_{k}}-M_{j_{k-1}}\right)\left(C_{j_{k}}-C_{j_{k-1}}\right) \\
& \quad<\left(M_{j_{k}}-M_{j_{k-1}}\right)\left(C_{j}-C_{j_{k}}+C_{j_{k}}-C_{j_{k-1}}\right)
\end{aligned}
$$




$$
\begin{aligned}
& \Leftrightarrow\left(M_{j}-M_{j_{k}}\right)\left(C_{j_{k}}-C_{j_{k-1}}\right)<\left(M_{j_{k}}-M_{j_{k-1}}\right)\left(C_{j}-C_{j_{k}}\right) \\
& \Leftrightarrow \frac{M_{j}-M_{j_{k}}}{C_{j}-C_{j_{k}}}<\frac{M_{j_{k}}-M_{j_{k-1}}}{C_{j_{k}}-C_{j_{k-1}}}=d_{k}^{*}(m) .
\end{aligned}
$$

Since this inequality holds for all $j>j_{k}$, and there are only finitely many indices $j$ greater than $j_{k}$, we have that

$$
d_{k+1}^{*}(m)=\max _{j>j_{k}} \frac{M_{j}-M_{j_{k}}}{C_{j}-C_{j_{k}}}<d_{k}^{*}(m) .
$$

Since $k \leq K(m)-1$ was arbitrary, the desired result follows.

It is easy to show that the $e_{k}^{*}$ are strictly decreasing in $k$.

Lemma 3.8 The $e_{k}^{*}, 1 \leq k \leq K$, as defined in Sect. 2.3.2, satisfy $e_{k+1}^{*}<e_{k}^{*}$ for $1 \leq k \leq K-1$.

Proof Fix $k \in\{1,2, \ldots, K-1\}$. We will show that $e_{k+1}^{*}<e_{k}^{*}$. By definition $e_{k}^{*} \geq e_{j}^{(k)}$ for all $j>j_{k-1}^{*}$, with strict inequality for all $j>j_{k}^{*}$. Hence, since $\rho_{i}$ is strictly decreasing in its argument, $\rho_{i}\left(e_{k}^{*}\right) \leq \rho_{i}\left(e_{j}^{(k)}\right)$ for $j>j_{k-1}^{*}$ and all $i$, with again, strict inequality if $j>j_{k}^{*}$. But this implies that for $j>j_{k}^{*}$,

$$
\sum_{i=j_{k-1}^{*}+1}^{j} \rho_{i}\left(e_{k}^{*}\right)<\sum_{i=j_{k-1}^{*}+1}^{j} \rho_{i}\left(e_{j}^{(k)}\right)=C_{j}-C_{j_{k-1}^{*}}
$$

where the equality follows from the definition of $e_{j}^{(k)}$. But, again by definition, for $j>j_{k}^{*}$,

$$
\begin{aligned}
\sum_{i=j_{k-1}^{*}+1}^{j} \rho_{i}\left(e_{k}^{*}\right) & =\sum_{i=j_{k-1}^{*}+1}^{j_{k}^{*}} \rho_{i}\left(e_{k}^{*}\right)+\sum_{i=j_{k}^{*}+1}^{j} \rho_{i}\left(e_{k}^{*}\right) \\
& =C_{j_{k}^{*}}-C_{j_{k-1}^{*}}+\sum_{i=j_{k}^{*}+1}^{j} \rho_{i}\left(e_{k}^{*}\right)
\end{aligned}
$$

and hence, we obtain

$$
\sum_{i=j_{k}^{*}+1}^{j} \rho_{i}\left(e_{k}^{*}\right)<C_{j}-C_{j_{k}^{*}}
$$




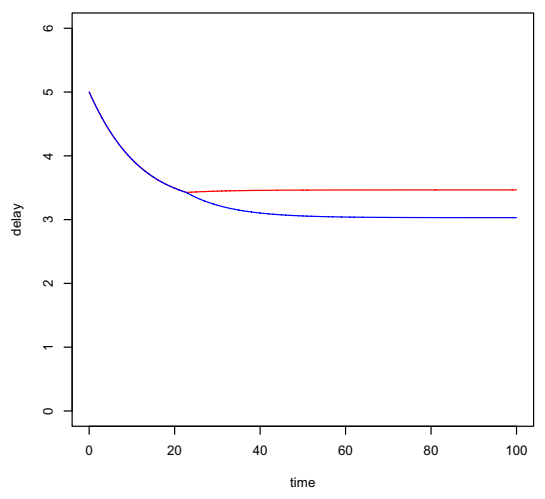

(a) Chokepoint at queue 1 in equilibrium

$$
\begin{gathered}
C_{1}=2, C_{2}=5 \\
\rho_{1}(d)=4 e^{-0.2 d}, \rho_{2}(d)=5.5 e^{-0.2 d}
\end{gathered}
$$

In both plots (a) and (b),

$$
\text { denotes delay at queue } 1, d_{1}(\cdot)
$$

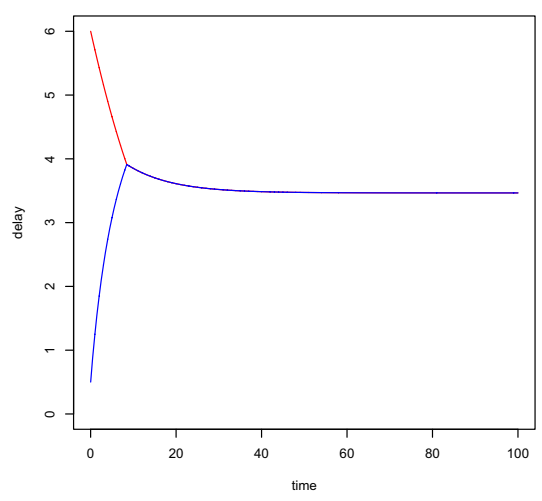

(b) Chokepoint at queue 2 in equilibrium

$$
\begin{gathered}
C_{1}=3, C_{2}=5 \\
\rho_{1}(d)=4 e^{-0.2 d}, \rho_{2}(d)=6 e^{-0.2 d}
\end{gathered}
$$

denotes delay at queue $2, d_{2}(\cdot)$

Fig. 2 Examples of trajectories for $d_{1}(\cdot)$ and $d_{2}(\cdot)$

Thus, since all of the $\rho_{i}$ are strictly decreasing in their arguments, for $j>j_{k}^{*}$, the solution, $e_{j}^{(k+1)}$, to

$$
\sum_{i=j_{k}^{*}+1}^{j} \rho_{i}(s)=C_{j}-C_{j_{k}^{*}}
$$

must satisfy $e_{j}^{(k+1)}<e_{k}^{*}$. Since this is true for all $j>j_{k}^{*}$, we have also immediately that $\max _{j>j_{k}^{*}} e_{j}^{(k+1)}<e_{k}^{*}$, that is, that $e_{k+1}^{*}<e_{k}^{*}$.

Figure 2 gives examples for a system with $N=2$, illustrating trajectories of $d_{1}$ and $d_{2}$ in two cases, the first where the equilibrium chokepoint $j_{1}^{*}$ is at 1 , and the second when it is at 2 .

\section{Proofs of Asymptotics of the Controlled System}

In this section we show that the minmax delay, $d_{1}^{*}(t)$, and first chokepoint, $j_{1}(t)$, converge to $e^{*}$ and $j^{*}$ respectively as $t \rightarrow \infty$.

Although the $j_{k}$ depend on time, to ease the notation in the following, we shall sometimes suppress this time dependence when $k>1$. 


\subsection{Preliminary Lemmas}

We begin this section by deriving some preliminary lemmas which will be used in the proof of the main theorem. The first lemma compares differences in delays at adjacent chokepoints with differences in the virtual delays. It also shows that the virtual delays are decreasing in $k$ and that the delay at each chokepoint, apart from the first, is strictly less than the virtual delay there. Recall that $K(t)$ is the number of chokepoints at time $t$.

Lemma 4.1 For each $t \geq 0$, if $K(t)>1$ then for $1 \leq k \leq K(t)-1$,

$$
\delta_{j_{k}(t)}(t)>\delta_{j_{k+1}(t)}(t)>d_{k+1}^{*}(t),
$$

and

$$
d_{k}^{*}(t)-d_{k+1}^{*}(t) \leq \frac{C_{j_{k+1}(t)}}{C_{j_{k+1}(t)}-C_{j_{k}(t)}}\left(\delta_{j_{k}(t)}(t)-\delta_{j_{k+1}(t)}(t)\right)
$$

where equality holds in (4.2) when $k=1$, and the inequality is strict for $k>1$.

Proof Observe first that for fixed $i$ and $j$ such that $1 \leq i<j \leq N$, we have

$$
\begin{aligned}
\delta_{j}(t) & =\frac{M_{j}(t)}{C_{j}}=\frac{M_{i}(t)+M_{j}(t)-M_{i}(t)}{C_{j}} \\
& =\frac{M_{i}(t)}{C_{i}} \frac{C_{i}}{C_{j}}+\frac{M_{j}(t)-M_{i}(t)}{C_{j}-C_{i}} \frac{C_{j}-C_{i}}{C_{j}} \\
& =\frac{C_{i}}{C_{j}} \delta_{i}(t)+\left(1-\frac{C_{i}}{C_{j}}\right) \frac{M_{j}(t)-M_{i}(t)}{C_{j}-C_{i}},
\end{aligned}
$$

a convex combination of $\delta_{i}(t)$ and $\frac{M_{j}(t)-M_{i}(t)}{C_{j}-C_{i}}$. In the particular case where $i=j_{k}$ and $j=j_{k+1}$, we therefore have for $k=1,2, \ldots, K(t)-1$,

$$
\delta_{j_{k+1}(t)}(t)=\frac{C_{j_{k}(t)}}{C_{j_{k+1}(t)}} \delta_{j_{k}(t)}(t)+\left(1-\frac{C_{j_{k}(t)}}{C_{j_{k+1}(t)}}\right) d_{k+1}^{*}(t) .
$$

We now show that (4.1) holds, by induction on $k$. Consider first the case $k=1$. From (4.4) above,

$$
\delta_{j_{2}(t)}(t)=\frac{C_{j_{1}(t)}}{C_{j_{2}(t)}} \delta_{j_{1}(t)}(t)+\left(1-\frac{C_{j_{1}(t)}}{C_{j_{2}(t)}}\right) d_{2}^{*}(t),
$$


a convex combination of $\delta_{j_{1}(t)}$ and $d_{2}^{*}(t)$. From Lemma 3.7, $d_{1}^{*}(t)>d_{2}^{*}(t)$, and hence, since $\delta_{j_{1}(t)}(t)=d_{1}^{*}(t)>d_{2}^{*}(t)$, it follows immediately that $\delta_{j_{2}(t)}(t)>d_{2}^{*}(t)$. On the other hand, applying the same inequality to $d_{2}^{*}(t)$ in $(4.5)$, we obtain $\delta_{j_{2}(t)}(t)<$ $\delta_{j_{1}(t)}(t)$. Thus (4.1) holds for $k=1$. Now assume that (4.1) holds for $k=n-1$ for some $n$ such that $2 \leq n \leq K(t)-1$. We will show that it holds for $k=n$ using very similar arguments to those for $k=1$. From (4.4) above,

$$
\delta_{j_{n+1}(t)}(t)=\frac{C_{j_{n}(t)}}{C_{j_{n+1}(t)}} \delta_{j_{n}(t)}(t)+\left(1-\frac{C_{j_{n}(t)}}{C_{j_{n+1}(t)}}\right) d_{n+1}^{*}(t) .
$$

By the induction hypothesis, $\delta_{j_{n}(t)}(t)>d_{n}^{*}(t)$, and, from Lemma 3.7, $d_{n}^{*}(t)>$ $d_{n+1}^{*}(t)$, thus implying $\delta_{j_{n}(t)}(t)>d_{n+1}^{*}(t)$. Using this in (4.6) we obtain $\delta_{j_{n}(t)}(t)>$ $\delta_{j_{n+1}(t)}(t)>d_{n+1}^{*}(t)$. This completes the induction step and it follows that (4.1) holds for each $k=1,2, \ldots, K(t)-1$.

We now show (4.2) holds. Using (4.4), we have

$$
\begin{aligned}
\delta_{j_{k}(t)}(t)-\delta_{j_{k+1}(t)}(t) & =\left(1-\frac{C_{j_{k}(t)}}{C_{j_{k+1}(t)}}\right)\left(\delta_{j_{k}(t)}(t)-d_{k+1}^{*}(t)\right) \\
& \geq\left(1-\frac{C_{j_{k}(t)}}{C_{j_{k+1}(t)}}\right)\left(d_{k}^{*}(t)-d_{k+1}^{*}(t)\right)
\end{aligned}
$$

where the inequality follows from the fact that $\delta_{j_{1}(t)}(t)=d_{1}^{*}(t)$ for $k=1$, and with $k-1$ in place of $k$ in (4.1) for $k \geq 2$. Furthermore, the inequality is strict unless $k=1$, in which case it is an equality.

The second lemma gives a bound on the derivative at regular points of the virtual delay for chokepoints.

Lemma 4.2 Let $K(t)$ be the number of chokepoints at time $t$. Then for $k$ such that $2 \leq k \leq K(t)$, at any regular point $t$,

$$
\dot{\delta}_{j_{k}(t)}(t)<\psi_{j_{k}(t)}\left(d_{k}^{*}(t)\right)-1
$$

Proof Fix $t$ and $k \in\{2,3, \ldots, K(t)\}$. We begin with the general expression for the derivative, from (3.3):

$$
\begin{aligned}
\dot{\delta}_{j_{k}(t)}(t) & =\frac{1}{C_{j_{k}(t)}} \sum_{i \leq j_{k}}\left[\rho_{i}\left(d_{i}(t)\right)-\Lambda_{i}(m(t))\right] \\
& =\frac{1}{C_{j_{k}(t)}}\left(\sum_{n=1}^{k} \sum_{i=j_{n-1}(t)+1}^{j_{n}(t)} \rho_{i}\left(d_{n}^{*}(t)\right)-C_{j_{k}(t)}\right) \\
& =\frac{1}{C_{j_{k}(t)}}\left(R_{1, j_{1}(t)}\left(d_{1}^{*}(t)\right)+\sum_{n=2}^{k}\left[R_{1, j_{n}(t)}\left(d_{n}^{*}(t)\right)-R_{1, j_{n-1}(t)}\left(d_{n}^{*}(t)\right)\right]\right)-1
\end{aligned}
$$




$$
\begin{aligned}
& =\frac{1}{C_{j_{k}(t)}}\left(C_{j_{1}(t)} \psi_{j_{1}(t)}\left(d_{1}^{*}(t)\right)+\sum_{n=2}^{k}\left[C_{j_{n}(t)} \psi_{j_{n}(t)}\left(d_{n}^{*}(t)\right)-C_{j_{n-1}(t)} \psi_{j_{n-1}(t)}\left(d_{n}^{*}(t)\right)\right]\right)-1 \\
& =\frac{1}{C_{j_{k}(t)}}\left(\sum_{n=1}^{k-1} C_{j_{n}(t)}\left[\psi_{j_{n}(t)}\left(d_{n}^{*}(t)\right)-\psi_{j_{n}(t)}\left(d_{n+1}^{*}(t)\right)\right]+C_{j_{k}(t)} \psi_{j_{k}(t)}\left(d_{k}^{*}(t)\right)\right)-1 \\
& <\psi_{j_{k}(t)}\left(d_{k}^{*}(t)\right)-1 .
\end{aligned}
$$

The second equality above follows from Item 1 of Proposition 2.3, which implies that $d_{i}(t)=d_{n}^{*}(t)$ for $j_{n-1}(t)<i \leq j_{n}(t)$, and the final inequality follows since $d_{n}^{*}(t)>d_{n+1}^{*}(t)$ implies $\psi_{j_{n}(t)}\left(d_{n}^{*}(t)\right)<\psi_{j_{n}(t)}\left(d_{n+1}^{*}(t)\right)$. The reasoning in (4.8) also holds for $k=1$, but then the final inequality is replaced by an equality.

\subsection{Asymptotics of $d_{1}^{*}(t)$ and of $j_{1}(t)$}

In this section, we prove Items 1 and 2 of Theorem 2.4. Note that in these proofs, we have reused the symbols $t_{k}$, i.e. these are not the same as in Theorem 2.5.

Proof of Items 1 and 2 of Theorem 2.4 In this argument, a regular point means a time $t>0$ that is a regular point for $m(\cdot)$ and $d_{1}^{*}(\cdot)$. Then almost every $t$ is such a regular point. Note that by Lemma 3.2, at such a $t, m_{i}(t)>0$ for $i=1,2, \ldots, N$.

Preliminaries on properties at regular points. Fix a regular point $t>0$. We claim that for any $j$ such that $\delta_{j}(t)=d_{1}^{*}(t)$, we have $\dot{\delta}_{j}(t)=\dot{d}_{1}^{*}(t)$. This is a well known result at regular points for maxima of Lipschitz functions, which can be seen as follows (cf. the justification of (3.2) in Dai and Weiss [6]):

$$
\begin{aligned}
& \dot{d}_{1}^{*}(t)=\lim _{s \uparrow t} \frac{d_{1}^{*}(s)-d_{1}^{*}(t)}{s-t} \leq \lim _{s \uparrow t} \frac{\delta_{j}(s)-\delta_{j}(t)}{s-t}=\dot{\delta}_{j}(t) \\
& \dot{d}_{1}^{*}(t)=\lim _{s \downarrow t} \frac{d_{1}^{*}(s)-d_{1}^{*}(t)}{s-t} \geq \lim _{s \downarrow t} \frac{\delta_{j}(s)-\delta_{j}(t)}{s-t}=\dot{\delta}_{j}(t),
\end{aligned}
$$

and so $\dot{d}_{1}^{*}(t)=\dot{\delta}_{j}(t)$. Here we have used the fact that $s<t$ in the first line to reverse the inequality $d_{1}^{*}(s) \geq \delta_{j}(s)$.

Inequalities for $d_{1}^{*}(t)$ and $\dot{\delta}_{j^{*}}(t)$ at a regular point. The signs of $\dot{d}_{1}^{*}(t)$ and $\dot{\delta}_{j^{*}}(t)$ depend on the position of $d_{1}^{*}(t)$ relative to $e^{*}$. We now derive inequalities that we will be using later under various conditions. Recall that $j_{1}(t)=\max \left\{j: \delta_{j}(t)=d_{1}^{*}(t)\right\}$. Suppose $t>0$ is a regular point. We consider three cases.

(Case $\left.d_{1}^{*}(t)>e^{*}\right) \quad$ If $d_{1}^{*}(t)>e^{*}$, then

$$
\begin{aligned}
\dot{d}_{1}^{*}(t) & =\dot{\delta}_{j_{1}(t)}(t) \\
& =\psi_{j_{1}(t)}\left(d_{1}^{*}(t)\right)-1<0
\end{aligned}
$$

where we have used Proposition 2.2, and Lemmas 3.2 and 3.4, for the second equality, and the facts that $d_{1}^{*}(t)>e^{*} \geq e_{j}$ and $\psi_{j}$ is a strictly decreasing function with $\psi_{j}\left(e_{j}\right)=1$ for each $j$, for the last inequality. 
(Case $\left.d_{1}^{*}(t)<e^{*}\right)$ If $d_{1}^{*}(t)<e^{*}$, then we have $d_{1}^{*}(t) \geq \delta_{j^{*}}(t)$, with

$$
\begin{aligned}
\dot{\delta}_{j^{*}}(t) & =\sum_{i \leq j^{*}}\left(\rho_{i}\left(d_{i}(t)\right)-\Lambda_{i}(m(t))\right) / C_{j^{*}} \\
& \geq \sum_{i \leq j^{*}}\left(\rho_{i}\left(d_{1}^{*}(t)\right)-\Lambda_{i}(m(t))\right) / C_{j^{*}} \\
& \geq \psi_{j^{*}}\left(d_{1}^{*}(t)\right)-1 \\
& >0
\end{aligned}
$$

where we used Lemma 3.4 for the first equality, in the second line we used the facts that $d_{i}(t) \leq d_{1}^{*}(t)$, and $\rho_{i}$ is a decreasing function for each $i$, and in the third line we used the definition of $\psi_{j^{*}}$, and the fact that $\sum_{i \leq j^{*}} \Lambda_{i}(m(t)) \leq C_{j^{*}}$. For the fourth line, we used the assumption that $d_{1}^{*}(t)<e^{*}=e_{j^{*}}$ and the fact that $\psi_{j^{*}}$ is a strictly decreasing function with $\psi_{j^{*}}\left(e_{j^{*}}\right)=1$.

(Case $d_{1}^{*}(t)=e^{*}$ ) If $d_{1}^{*}(t)=e^{*}$, we have that $d_{1}^{*}(t) \geq \delta_{j^{*}}(t)$, and, proceeding exactly as in (4.10), except that in the last line there is now equality, we have:

$$
\dot{\delta}_{j^{*}}(t) \geq \psi_{j^{*}}\left(d_{1}^{*}(t)\right)-1=0
$$

Also, the first inequality is an equality if $j_{1}(t)=j^{*}$.

Proof of Item 1 of Theorem 2.4 We again must consider cases, this time two.

(Case $\left.d_{1}^{*}(0)>e^{*}\right) \quad$ Here it follows from the fact that (4.9) holds for an arbitrary regular point $t>0$ for which $d_{1}^{*}(t)>e^{*}$ that $d_{1}^{*}(\cdot)$ is strictly decreasing on $[0, \zeta)$, where

$$
\zeta=\inf \left\{t \geq 0: d_{1}^{*}(t)=e^{*}\right\}
$$

If $\zeta=+\infty$, then $d_{1}^{*}(t) \rightarrow e^{*}$ as $t \rightarrow \infty$. This can be seen by arguing by contradiction as follows. If $d_{1}^{*}(t) \nrightarrow e^{*}$, then using the aforementioned decreasing property of $d_{1}^{*}(\cdot)$, there is $\epsilon>0$ such that $d_{1}^{*}(t)>e^{*}+\epsilon$ for all $t \geq 0$. But by (4.9), using the fact that $\psi_{j}$ is a strictly decreasing function for each $j$, we would then have that

$$
\begin{aligned}
d_{1}^{*}(t) & =d_{1}^{*}(0)+\int_{0}^{t}\left(\psi_{j_{1}(s)}\left(d_{1}^{*}(s)\right)-1\right) d s \\
& \leq d_{1}^{*}(0)+\int_{0}^{t}\left(\psi_{j_{1}(s)}\left(e^{*}+\epsilon\right)-1\right) d s \\
& \leq d_{1}^{*}(0)+t\left(\max _{j} \psi_{j}\left(e^{*}+\epsilon\right)-1\right) .
\end{aligned}
$$

Since $\psi_{j}\left(e^{*}+\epsilon\right)<\psi_{j}\left(e^{*}\right) \leq 1$ for each $j$, the maximum in the above is strictly less than one and it would follow that $d_{1}^{*}(t)=e^{*}$ for some $t \leq\left(d_{1}^{*}(0)-e^{*}\right) /(1-$ $\max _{j} \psi_{j}\left(e^{*}+\epsilon\right)$ ), which contradicts the assumption that $\zeta=+\infty$. Thus, Item 1 is proved for $d_{1}^{*}(0)>e^{*}$ if $\zeta=+\infty$. Now, with the same initial condition, if $\zeta<\infty$, 
then using (4.9), we see that $d_{1}^{*}(t) \leq e^{*}$ for all $t \geq \zeta$. Thus, in this case, the asymptotic behavior of $d_{1}^{*}(\cdot)$ reduces to that for $d_{1}^{*}(0)=e^{*}$.

(Case $\left.d_{1}^{*}(0) \leq e^{*}\right)$ If $d_{1}^{*}(t)=e^{*}$ for all $t \geq 0$, then Item 1 is proved. If $d_{1}^{*}(\cdot) \not \equiv e^{*}$ then from the above, we have that $d_{1}^{*}(t) \leq e^{*}$ for all $t \geq 0$ and there is a time $t_{1}>0$ such that $d_{1}^{*}\left(t_{1}\right)<e^{*}$. Define $\check{e}^{*}=\max \left\{e_{j}: e_{j}<e^{*}\right\}$ to be the second greatest value that the $e_{j}$ take, let $\gamma=\max _{j^{*} \leq j<i \leq N} \frac{C_{j}}{C_{i}}$, and set $e^{\dagger}=\gamma e^{*}+(1-\gamma) \check{e}^{*}$. Note that $e^{\dagger}>\check{e}^{*}$ and set $\ell^{*}=\frac{1}{2}\left(e^{*}+e^{\dagger}\right)$. Then $\ell^{*}>e^{\dagger}$.

Let

$$
t_{2}=\inf \left\{t \geq t_{1}: d_{1}^{*}(t) \leq \ell^{*} \text { or } d_{1}^{*}(t)=\delta_{j^{*}}(t)\right\} .
$$

Then $t_{2}<\infty$, since at any regular point $t$ in $\left[t_{1}, t_{2}\right)$,

$$
\begin{aligned}
\dot{d}_{1}^{*}(t)=\dot{\delta}_{j_{1}(t)}(t) & =\psi_{j_{1}(t)}\left(d_{1}^{*}(t)\right)-1 \quad \text { where } j_{1}(t) \neq j^{*} \\
& \leq \psi_{j_{1}(t)}\left(\ell^{*}\right)-1 \\
& <0 \quad \text { since } \ell^{*}>\check{e}^{*}=\max _{j \neq j^{*}} e_{j}
\end{aligned}
$$

and by (4.10)-(4.11),

$$
\dot{\delta}_{j^{*}}(t) \geq 0
$$

and so if $d_{1}^{*}(t)>\ell^{*}$ on $\left[t_{1}, \infty\right)$, then $d_{1}^{*}=\delta_{j^{*}}$ at, or before, the time

$$
t_{1}+\frac{e^{*}-\delta_{j^{*}}\left(t_{1}\right)}{1-\max _{j \neq j^{*}} \psi_{j}\left(\ell^{*}\right)},
$$

and so $t_{2}<\infty$.

Now let

$$
t_{3}=\inf \left\{t \geq t_{2}: d_{1}^{*}(t)=\delta_{j^{*}}(t)\right\}
$$

If $t_{2}=t_{3}$, then $t_{3}$ is finite, otherwise, if $t_{2}<t_{3}$, then

$$
d_{1}^{*}(t) \leq \ell^{*} \text { for all } t \in\left[t_{2}, t_{3}\right) .
$$

This is because when $t_{2}<t_{3}, d_{1}^{*}\left(t_{2}\right) \leq \ell^{*}$ and at a regular point $t$ in $\left[t_{2}, t_{3}\right)$ for which $d_{1}^{*}(t)>\check{e}^{*}$, we have

$$
\begin{aligned}
\dot{d}_{1}^{*}(t)=\dot{\delta}_{j_{1}(t)}(t) & =\psi_{j_{1}(t)}\left(d_{1}^{*}(t)\right)-1 \quad \text { for some } j_{1}(t) \neq j^{*} \\
& \leq 0 \quad \text { since } e_{j_{1}(t)} \leq \check{e}^{*}
\end{aligned}
$$

and so $d_{1}^{*}$ is non-increasing on $\left\{t \in\left[t_{2}, t_{3}\right): d_{1}^{*}(t)>\check{e}^{*}\right\}$. 
Given (4.17), by (4.10), at regular points $t \in\left[t_{2}, t_{3}\right)$ we have

$$
\begin{aligned}
\dot{\delta}_{j^{*}}(t) & \geq \psi_{j^{*}}\left(d_{1}^{*}(t)\right)-1 \\
& \geq \psi_{j^{*}}\left(\ell^{*}\right)-1>0
\end{aligned}
$$

and so

$$
t_{3} \leq t_{2}+\frac{\ell^{*}-\delta_{j^{*}}\left(t_{2}\right)}{\psi_{j^{*}}\left(\ell^{*}\right)-1}<\infty .
$$

Thus, in any event, $t_{3}$ is finite.

Now for $t \in\left[t_{3}, \infty\right), d_{1}^{*}(t) \geq \delta_{j^{*}}(t)$, where by (4.10), if $t$ is a regular point then

$$
\dot{\delta}_{j^{*}}(t) \geq \psi_{j^{*}}\left(d_{1}^{*}(t)\right)-1>0 \text { if } d_{1}^{*}(t)<e^{*},
$$

and so

$$
d_{1}^{*}(t) \geq \delta_{j^{*}}(t) \geq \delta_{j^{*}}\left(t_{3}\right)=d_{1}^{*}\left(t_{3}\right) \text { for all } t \in\left[t_{3}, \infty\right) .
$$

It also follows from the above that $t_{4}=\inf \left\{t \geq t_{3}: d_{1}^{*}(t) \geq \ell^{*}\right\}$ is finite and that

$$
d_{1}^{*}\left(t_{4}\right)=\delta_{j^{*}}\left(t_{4}\right)
$$

To see the finiteness of $t_{4}$, note that for $t \in\left[t_{3}, t_{4}\right)$ that is a regular point, from (4.18) we have

$$
\begin{aligned}
\dot{\delta}_{j^{*}}(t) & \geq \psi_{j^{*}}\left(d_{1}^{*}(t)\right)-1 \\
& \geq \psi_{j^{*}}\left(\ell^{*}\right)-1>0
\end{aligned}
$$

and $d_{1}^{*}(t) \geq \delta_{j^{*}}(t)$ for all $t \in\left[t_{3}, t_{4}\right)$, so that for $t_{3}<t_{4}$,

$$
t_{4} \leq t_{3}+\frac{\ell^{*}-\delta_{j^{*}}\left(t_{3}\right)}{\psi_{j^{*}}\left(\ell^{*}\right)-1}<\infty .
$$

For the other claim (4.20), note that if $t_{4}=t_{3}$, then the claim holds. On the other hand, if $t_{3}<t_{4}$, then $d_{1}^{*}\left(t_{3}\right)<\ell^{*}$, and for a proof by contradiction suppose that $d_{1}^{*}\left(t_{4}\right)>\delta_{j^{*}}\left(t_{4}\right)$. Then by the continuity of $d_{1}^{*}$ and $\delta_{j^{*}}$ there is $\epsilon>0$ such that $t_{3}<t_{4}-\epsilon, d_{1}^{*}(t)>\delta_{j^{*}}(t)$ and $d_{1}^{*}(t)>\check{e}^{*}$ for all $t \in\left(t_{4}-\epsilon, t_{4}+\epsilon\right)$. Then for any regular point $t \in\left(t_{4}-\epsilon, t_{4}+\epsilon\right)$

$$
\begin{aligned}
\dot{d}_{1}^{*}(t)=\dot{\delta}_{j_{1}(t)}(t) & =\psi_{j_{1}(t)}\left(d_{1}^{*}(t)\right)-1 \quad \text { where } j_{1}(t) \neq j^{*} \\
& <0 \quad \text { since } d_{1}^{*}(t)>\check{e}^{*}=\max _{j \neq j^{*}} e_{j} .
\end{aligned}
$$

If follows that $d_{1}^{*}\left(t_{4}\right)<d_{1}^{*}\left(t_{4}-\epsilon\right)<\ell^{*}$ by the definition of $t_{4}$ when $t_{4}>t_{3}$, but this contradicts the fact that $d_{1}^{*}\left(t_{4}\right) \geq \ell^{*}$. 
Finally, let $t_{5}=\inf \left\{t \geq t_{4}: d_{1}^{*}(t)=e^{*}\right\}$. Then by a similar argument to that for (4.19), we have that $d_{1}^{*}(t) \geq \ell^{*}$ and $\delta_{j^{*}}(t) \geq \ell^{*}$ for all $t \in\left[t_{4}, t_{5}\right)$. Also, by a similar argument to that for (4.20), $d_{1}^{*}(t)=\delta_{j^{*}}(t)$ for each $t \in\left[t_{4}, t_{5}\right)$. Indeed, if there is $t \in\left(t_{4}, t_{5}\right)$ such that $d_{1}^{*}(t)>\delta_{j}^{*}(t)$, then for $t_{\dagger}=\sup \left\{s \in\left(t_{4}, t\right): d_{1}^{*}(s)=\delta_{j^{*}}(s)\right\}$, we have, for regular points $s \in\left(t_{\dagger}, t\right), \dot{d}_{1}^{*}(s)<0$ as in (4.21), and $\dot{\delta}_{j^{*}}(s)>0$ by (4.10), and so $d_{1}^{*}(\cdot)-\delta_{j^{*}}(\cdot)$ is decreasing on $\left(t_{\dagger}, t\right)$. But this contradicts $d_{1}^{*}(t)>\delta_{j^{*}}(t)$. Thus for a regular point $t \in\left[t_{4}, t_{5}\right)$,

$$
\begin{aligned}
\dot{d}_{1}^{*}(t) & =\dot{\delta}_{j^{*}}(t) \\
& =\psi_{j^{*}}\left(d_{1}^{*}(t)\right)-1>0 .
\end{aligned}
$$

It then follows by a similar argument to that given for the case $d_{1}^{*}(0)>e^{*}$ that either $t_{5}=+\infty$ and $d_{1}^{*}(t) \rightarrow e^{*}$ from below as $t \rightarrow \infty$, or $t_{5}<\infty$ and $d_{1}^{*}\left(t_{5}\right)=e^{*}$. In the latter case, $d_{1}^{*}(t)=e^{*}$ for all $t \geq t_{5}$ because $e^{*} \geq d_{1}^{*}(t) \geq \delta_{j^{*}}(t)$, for $t \geq t_{5}$, and by (4.10) and (4.11), $\delta_{j^{*}}(\cdot)$ is non-decreasing on $\left[t_{5}, \infty\right)$, and so $\delta_{j^{*}}(t)=d_{1}^{*}(t)=e^{*}$ for all $t \geq t_{5}$. Item 1 is now proved.

Proof of Item 2 of Theorem 2.4 Again, we consider the two cases, $d_{1}^{*}(0)>e^{*}$ and $d_{1}^{*}(0) \leq e^{*}$.

(Case $\left.d_{1}^{*}(0) \leq e^{*}\right) \quad$ We will show that if $d_{1}^{*}(0) \leq e^{*}$ then $j_{1}(t)=j^{*}$ for $t>t_{4}$ where $t_{4}$ is as defined in the proof of Item 1. Observe first that since $\delta_{j^{*}}(t)=d_{1}^{*}(t)$ for all $t \geq t_{4}$, we immediately have $j_{1}(t) \geq j^{*}$ for all $t \geq t_{4}$. Thus the case when $j^{*}=N$ is proved.

Now suppose that $j^{*}<N$. We need only show that $j_{1}(t)<j^{*}+1$ for all sufficiently large $t>t_{4}$.

We showed in Item 1 that

$$
d_{1}^{*}(t)=\delta_{j^{*}}(t) \geq \ell^{*} \text { for all } t \geq t_{4},
$$

and at any regular point $t \geq t_{4}, \dot{d}_{1}^{*}(t)=\dot{\delta}_{j^{*}}(t) \geq 0$ where the last inequality is by (4.10)-(4.11) and is strict if $d_{1}^{*}(t)<e^{*}$.

Let $t_{6}=t_{4}+1$. We show first that for $t \geq t_{6}$, we have $K(t) \geq 2$, that is, there are at least two chokepoints. (Here $t_{6}$ could be replaced by any other fixed time greater than $t_{4}$.) For a proof by contradiction, suppose that there is $t^{\prime} \geq t_{6}$ such that $d_{1}^{*}\left(t^{\prime}\right)=\delta_{N}\left(t^{\prime}\right) \geq \ell^{*}$. Then by continuity of both $\delta_{N}(\cdot)$ and $d_{1}^{*}(\cdot)$, in a neighbourhood of $t^{\prime}, d_{N}(\cdot)$ will be close to $d_{1}^{*}(\cdot)$. Specifically, from Lemma 4.1 we have for any $t$,

$$
\begin{aligned}
d_{1}^{*}(t)-d_{N}(t) & =d_{1}^{*}(t)-d_{2}^{*}(t)+d_{2}^{*}(t)-\cdots-d_{K(t)-1}^{*}(t)+d_{K(t)-1}^{*}(t)-d_{K(t)}^{*}(t) \\
& \leq \sum_{i=1}^{K(t)-1} \frac{C_{j_{i+1}(t)}}{C_{j_{i+1}(t)}-C_{j_{i}(t)}}\left(\delta_{j_{i}(t)}(t)-\delta_{j_{i+1}(t)}(t)\right) \\
& \leq\left(\max _{j^{*} \leq n_{1}<n_{2} \leq N} \frac{C_{n_{2}}}{C_{n_{2}}-C_{n_{1}}}\right) \sum_{i=1}^{K(t)-1}\left(\delta_{j_{i}(t)}(t)-\delta_{j_{i+1}(t)}(t)\right)
\end{aligned}
$$




$$
\begin{aligned}
& =\left(\max _{j^{*} \leq n_{1}<n_{2} \leq N} \frac{C_{n_{2}}}{C_{n_{2}}-C_{n_{1}}}\right)\left(\delta_{j_{1}(t)}(t)-\delta_{N}(t)\right) \\
& =\left(\max _{j^{*} \leq n_{1}<n_{2} \leq N} \frac{C_{n_{2}}}{C_{n_{2}}-C_{n_{1}}}\right)\left(d_{1}^{*}(t)-\delta_{N}(t)\right) .
\end{aligned}
$$

Then by the continuity of $\delta_{N}(\cdot)$ and $d_{1}^{*}(\cdot)$, there exists $1>\epsilon>0$ such that for all $s \in\left(t^{\prime}-\epsilon, t^{\prime}+\epsilon\right)$, we have $d_{1}^{*}(s)-\delta_{N}(s)<\left(\ell^{*}-e^{\dagger}\right) /\left(\max _{j^{*} \leq n_{1}<n_{2} \leq N} \frac{C_{n_{2}}}{C_{n_{2}}-C_{n_{1}}}\right)$, and hence by (4.22) we have, $d_{1}^{*}(s)-d_{N}(s) \leq \ell^{*}-e^{\dagger}$. Since $s \geq t_{4}, d_{1}^{*}(s) \geq \ell^{*}$, and this implies $d_{N}(s) \geq e^{\dagger}$. Now, since $N$ is the last chokepoint, by Lemmas 3.4 and 3.7, the decreasing property of the $\rho_{i}$, and since $e^{\dagger}>e_{N}$, we have that at any regular point $s \in\left(t^{\prime}-\epsilon, t^{\prime}+\epsilon\right), \dot{\delta}_{N}(s) \leq \psi_{N}\left(d_{N}(s)\right)-1<0$. So $\delta_{N}\left(t^{\prime}-\frac{1}{2} \epsilon\right)>\delta_{N}\left(t^{\prime}\right)=d_{1}^{*}\left(t^{\prime}\right)$. But since $\dot{d}_{1}^{*}(t) \geq 0$ for any regular point $t \geq t_{4}$, we also have $d_{1}^{*}\left(t^{\prime}-\frac{1}{2} \epsilon\right) \leq d_{1}^{*}\left(t^{\prime}\right)<$ $\delta_{N}\left(t^{\prime}-\frac{1}{2} \epsilon\right)$, which yields a contradiction, since by definition, $d_{1}^{*}(t) \geq \delta_{j}(t)$ for all $t$, and $1 \leq j \leq N$. Hence $\delta_{N}(t)<d_{1}^{*}(t)$ for all $t \geq t_{6}$, and also $K(t) \geq 2$ for all $t \geq t_{6}$. Observe that if $j^{*}=N-1$, then it also immediately follows that $j_{1}(t)=j^{*}$ for all $t \geq t_{6}$, so for the remainder of the argument below we assume that $j^{*}<N-1$.

Now consider $d_{2}^{*}(t)$ for $t \geq t_{6}$, noting that since $K(t) \geq 2$ for all $t \geq t_{6}$, the existence of $d_{2}^{*}(t)$ is guaranteed. We also note that $j_{2}(t)>j_{1}(t) \geq j^{*}$ for all $t \geq t_{6}$. From Lemma 4.1, noting that we have equality in that lemma for the case $k=1$, we have for $t \geq 0$,

$$
\begin{aligned}
d_{2}^{*}(t) & =d_{1}^{*}(t)-\frac{C_{j_{2}(t)}}{C_{j_{2}(t)}-C_{j_{1}(t)}}\left(\delta_{j_{1}(t)}(t)-\delta_{j_{2}(t)}(t)\right) \\
& =d_{1}^{*}(t)-\frac{C_{j_{2}(t)}}{C_{j_{2}(t)}-C_{j_{1}(t)}}\left(d_{1}^{*}(t)-\delta_{j_{2}(t)}(t)\right) \\
& =\frac{C_{j_{2}(t)}}{C_{j_{2}(t)}-C_{j_{1}(t)}} \delta_{j_{2}(t)}(t)-\frac{C_{j_{1}(t)}}{C_{j_{2}(t)}-C_{j_{1}(t)}} d_{1}^{*}(t)
\end{aligned}
$$

where the second equality follows since $d_{1}^{*}(t)=\delta_{j_{1}(t)}(t)$, and the third involves a simple rearrangement of the terms. Hence

$$
d_{2}^{*}(t) \geq \check{e}^{*} \Longleftrightarrow \delta_{j_{2}(t)}(t) \geq\left(1-\frac{C_{j_{1}(t)}}{C_{j_{2}(t)}}\right) \check{e}^{*}+\frac{C_{j_{1}(t)}}{C_{j_{2}(t)}} d_{1}^{*}(t) .
$$

Observe that for $t \geq t_{6}$ we have $e^{*} \geq d_{1}^{*}(t) \geq \ell^{*}>\check{e}^{*}$, so that if for any $t \geq t_{6}$, we have $\delta_{j_{2}(t)}(t)>e^{\dagger}$ we also have

$$
\begin{aligned}
\delta_{j_{2}(t)}(t) & >e^{\dagger} \\
& \geq \frac{C_{j_{1}(t)}}{C_{j_{2}(t)}} e^{*}+\left(1-\frac{C_{j_{1}(t)}}{C_{j_{2}(t)}}\right) \check{e}^{*} \\
& \geq \frac{C_{j_{1}(t)}}{C_{j_{2}(t)}} d_{1}^{*}(t)+\left(1-\frac{C_{j_{1}(t)}}{C_{j_{2}(t)}}\right) \check{e}^{*},
\end{aligned}
$$


the second inequality following from $\frac{C_{j_{1}(t)}}{C_{j_{2}(t)}} \leq \max _{j^{*} \leq j<i \leq N} \frac{C_{j}}{C_{i}}=\gamma$ and $e^{*}>\check{e}^{*}$, and the third inequality following since $d_{1}^{*}(t) \leq e^{*}$. Thus if $\delta_{j_{2}(t)}(t)>e^{\dagger}$ then the right hand inequality in (4.23) is satisfied and hence $d_{2}^{*}(t) \geq \check{e}^{*}$.

Note that at regular points $t \geq t_{6}, d_{1}^{*}(t) \geq \ell^{*}>\max _{j \neq j^{*}} e_{j}$ and $\dot{d}_{1}^{*}(t)=\dot{\delta}_{j_{1}(t)}(t)=$ $\psi_{j_{1}(t)}\left(d_{1}^{*}(t)\right)-1<0$ if $j_{1}(t) \neq j^{*}$. But $\dot{d}_{1}^{*}(t) \geq 0$ at $t \geq t_{6}$ that are regular points. It follows that we must have that $j_{1}(t)=j^{*}$ for all regular points that satisfy $t \geq t_{6}$. Below we will show that there is $t_{7} \geq t_{6}$ such that this also holds true for all non-regular points $t \geq t_{7}$.

Now for $t \geq t_{6}$, we consider $\delta_{j_{2}(t)}(t)$, where we know that $j_{2}(t)>j^{*}$. At any regular point $t \geq t_{6}$, if $\delta_{j_{2}(t)}(t)>e^{\dagger}$, then from the arguments above, this implies $d_{2}^{*}(t) \geq \check{e}^{*}$, and then using Lemma 4.2 we have $\dot{\delta}_{j_{2}(t)}(t)<\psi_{j_{2}(t)}\left(d_{2}^{*}(t)\right)-1 \leq \max _{j>j^{*}} \psi_{j}\left(\check{e}^{*}\right)-$ $1<0$. Set $t_{7}=\inf \left\{t \geq t_{6}: \delta_{j_{2}(t)}(t) \leq \frac{1}{2}\left(e^{\dagger}+\ell^{*}\right)\right\}$. Then in a similar fashion to proofs we have seen already, $t_{7}$ is finite. Moreover, $\delta_{j_{2}(t)}(t) \leq \frac{1}{2}\left(e^{\dagger}+\ell^{*}\right)<d_{1}^{*}(t)$ for $t \geq t_{7}$.

Then, by (4.1) with $k=1$, we have

$$
d_{2}^{*}(t)<\delta_{j_{2}(t)}(t) \leq \frac{e^{\dagger}+\ell^{*}}{2}<d_{1}^{*}(t) \text { for all } t \geq t_{7}
$$

Also, by (4.3) with $i=j^{*}$, for $j>j^{*}$ and any regular point $t \geq t_{7}$, since $j_{1}(t)=j^{*}$ at such a regular point, we have

$$
\begin{aligned}
\delta_{j}(t) & =\frac{C_{j^{*}}}{C_{j}} \delta_{j^{*}}(t)+\left(1-\frac{C_{j^{*}}}{C_{j}}\right) \frac{M_{j}(t)-M_{j^{*}}(t)}{C_{j}-C_{j^{*}}} \\
& \leq \frac{C_{j^{*}}}{C_{j}} d_{1}^{*}(t)+\left(1-\frac{C_{j^{*}}}{C_{j}}\right) d_{2}^{*}(t) \\
& \leq \frac{C_{j^{*}}}{C_{j}} d_{1}^{*}(t)+\left(1-\frac{C_{j^{*}}}{C_{j}}\right)\left(\frac{e^{\dagger}+\ell^{*}}{2}\right)
\end{aligned}
$$

where we used the definition of $d_{2}^{*}(t)$ for the first inequality and (4.24) for the second inequality. Then, using (4.25) and the fact that $d_{1}^{*}(t) \geq \ell^{*}$, we have

$$
\begin{aligned}
d_{1}^{*}(t)-\delta_{j}(t) & \geq\left(1-\frac{C_{j^{*}}}{C_{j}}\right)\left(d_{1}^{*}(t)-\frac{e^{\dagger}+\ell^{*}}{2}\right) \\
& \geq \min _{j>j^{*}}\left(1-\frac{C_{j^{*}}}{C_{j}}\right)\left(\frac{\ell^{*}-e^{\dagger}}{2}\right)>0 .
\end{aligned}
$$

Now the set of regular points is dense in $\left[t_{7}, \infty\right)$ and so it follows by the continuity of $d_{1}^{*}$ and $\delta_{j}$ that the above also holds for all $t \geq t_{7}$ for all $j>j^{*}$. It follows from this that $j_{1}(t)=j^{*}$ for all $t \geq t_{7}$.

(Case $d_{1}^{*}(0)>e^{*}$ ) When proving Item 1 of the theorem for $d_{1}^{*}(0)>e^{*}$, we defined $\zeta=\inf \left\{t \geq 0: d_{1}^{*}(t)=e^{*}\right\}$, in (4.12), and noted that if $\zeta<\infty$, then the asymptotic behavior of $d_{1}^{*}(\cdot)$ reduces to its asymptotic behavior when $d_{1}^{*}(0)=e^{*}$. This is also true 
of the asymptotic behavior of $j_{1}(t)$, which is again covered by the case $d_{1}^{*}(0) \leq e^{*}$. So it remains only to consider the case where $\zeta=+\infty$.

In the following, we assume $\zeta=+\infty$, that is $d_{1}^{*}(t)>e^{*}$ for all $t \geq 0$. We show first that, for any $\epsilon \in\left(0, d_{1}^{*}(0)-e^{*}\right), d_{1}^{*}(\cdot)$ reaches $e^{*}+\epsilon$ by some finite time $t_{\epsilon}$ and stays less than or equal to this level after $t_{\epsilon}$. This follows from the fact that $d_{1}^{*}(t) \rightarrow e^{*}$ as $t \rightarrow \infty$, and by (4.9), $d_{1}^{*}$ is monotone decreasing when $\zeta=+\infty$. We also have that $j_{1}(t)$ reaches $j^{*}$ in finite time. To see this, observe that if $j_{1}(t) \neq j^{*}$ for all $t \geq 0$, then $\max _{j \neq j^{*}} \psi_{j}\left(e^{*}\right)=\beta<1$, and then, using the monotonicity of $\psi_{j}(\cdot)$ and (4.9), we have at any regular point $t$ that

$$
\begin{aligned}
\dot{d}_{1}^{*}(t) & =\psi_{j_{1}(t)}\left(d_{1}^{*}(t)\right)-1 \\
& \leq \psi_{j_{1}(t)}\left(e^{*}\right)-1 \leq \beta-1<0,
\end{aligned}
$$

and so for some $t \leq\left(d_{1}^{*}(0)-e^{*}\right) /(1-\beta)$, we have that $d_{1}^{*}(t)=e^{*}$. But this contradicts the assumption that $\zeta=+\infty$ and so we must have that $j_{1}(t)=j^{*}$ for some $t$ sufficiently large. We need to now show that there is such a $t$ for which $j_{1}$ remains at $j^{*}$ ever after that time. We will do this by showing, using a proof by induction, that for any $j \neq j^{*}$, there is a $\eta>0$ and finite time after which $\delta_{j}(\cdot)$ is less than or equal to $e^{*}-\eta$ for all time.

If $j^{*}=N$, let $0<\eta<\frac{1}{2}(1-\theta) e^{*}$ where $\theta=\max _{j \neq j^{*}} \psi_{j}\left(\ell^{*}\right)$. If $j^{*}<N$, let $0<\eta<\frac{1}{2} \min \left(\alpha,(1-\theta) e^{*}\right)$ where

$$
\alpha=\frac{e^{*}-\ell^{*}}{\max _{j^{*} \leq i<j \leq N} \frac{C_{j}}{C_{j}-C_{i}}} .
$$

Note that $\beta=\max _{j \neq j^{*}} \psi_{j}\left(e^{*}\right)<\theta$. Then for all $\epsilon \in\left(0, \min \left(d_{1}^{*}(0)-e^{*}, \eta,\left(e^{*}(1-\right.\right.\right.$ $\theta)-\eta) / 2)$ ), we have $1-\theta-\frac{\eta+2 \epsilon}{e^{*}+\epsilon}>0$ and hence also $1-\beta-\frac{\eta+2 \epsilon}{e^{*}+\epsilon}>0$.

Fix such an $\epsilon>0$. We begin by deriving some general inequalities that we will use repeatedly in the proof. If $j_{1}(t)>1$, then for any $k \neq j^{*}, k<j_{1}(t)$ and any regular point $t \geq t_{\epsilon}$, if $\delta_{k}(t)>e^{*}-\eta$, then by Lemma 3.4,

$$
\begin{aligned}
\dot{\delta}_{k}(t) & =\frac{1}{C_{k}} \sum_{i \leq k}\left(\rho_{i}\left(d_{i}(t)\right)-\Lambda_{i}(m(t))\right)=\frac{1}{C_{k}} \sum_{i \leq k}\left(\rho_{i}\left(d_{1}^{*}(t)\right)-\frac{m_{i}(t)}{d_{1}^{*}(t)}\right) \\
& =\psi_{k}\left(d_{1}^{*}(t)\right)-\frac{M_{k}(t)}{C_{k} d_{1}^{*}(t)}=\psi_{k}\left(d_{1}^{*}(t)\right)-\frac{\delta_{k}(t)}{d_{1}^{*}(t)} \\
& \leq \psi_{k}\left(e^{*}\right)-\frac{e^{*}-\eta}{e^{*}+\epsilon} \\
& =\psi_{k}\left(e^{*}\right)-1+\frac{\eta+\epsilon}{e^{*}+\epsilon}<0 .
\end{aligned}
$$

where the equalities follow from Proposition 2.2, (2.17) and (3.2), and for the inequalities we have used the facts that $e^{*}-\eta<\delta_{k}(t), e^{*}<d_{1}^{*}(t) \leq e^{*}+\epsilon$, and $t \geq t_{\epsilon}$. Also, for any regular point $t \geq t_{\epsilon}$, since $d_{1}^{*}(t)>e^{*}$, we have, by (4.9), $\dot{\delta}_{j_{1}(t)}(t)=\dot{d}_{1}^{*}(t)=\psi_{j_{1}(t)}\left(d_{1}^{*}(t)\right)-1<0$. 
We are now ready to show, using a proof by induction on $j$, that if $j^{*}>1$, then for any $j<j^{*}$, there is a finite time (greater than or equal to $t_{\epsilon}$ ) after which $\delta_{j}$ is always less than or equal to $e^{*}-\eta$.

Suppose $j^{*}>1$ and $j<j^{*}$. We first consider $j=1$. Let $\tau_{1}=\inf \left\{t \geq t_{\epsilon}\right.$ : $\left.\delta_{1}(t) \leq e^{*}-\eta\right\}$. If $\tau_{1}=t_{\epsilon}$, then $\delta_{1}\left(\tau_{1}\right) \leq e^{*}-\eta$. Suppose that $\tau_{1}>t_{\epsilon}$ and $t$ is a regular point such that $t_{\epsilon} \leq t<\tau_{1}$. Then, $\delta_{1}(t)>e^{*}-\eta$. If $j_{1}(t)=1$, then by (4.9), $\dot{\delta}_{1}(t)=\dot{d}_{1}^{*}(t)=\psi_{1}\left(d_{1}^{*}(t)\right)-1 \leq \psi_{1}\left(e^{*}\right)-1<0$. On the other hand, if $j_{1}(t)>1$, then by (4.28),

$$
\dot{\delta}_{1}(t) \leq \psi_{1}\left(e^{*}\right)-1+\frac{\eta+\epsilon}{e^{*}+\epsilon}<0
$$

Thus, we have

$$
\tau_{1} \leq t_{\epsilon}+\frac{\epsilon+\eta}{1-\psi_{1}\left(e^{*}\right)-\frac{\eta+\epsilon}{e^{*}+\epsilon}}<+\infty
$$

Furthermore, for any regular point $t \geq \tau_{1}$ for which $\delta_{1}(t) \in\left(e^{*}-\eta, e^{*}\right.$, by the same reasoning as for (4.28), we have $\dot{\delta}_{1}(t)<0$. It then follows that $\delta_{1}(t) \leq e^{*}-\eta$ for all $t \geq \tau_{1}$, and we have shown the case $j=1$.

Now, for the induction step, fix $1<j<j^{*}$. Suppose that it has been shown that there are finite times $t_{\epsilon} \leq \tau_{1}<\cdots<\tau_{j-1}$ such that $\delta_{i}(t) \leq e^{*}-\eta$ for all $t \geq \tau_{i}$, for $i=1, \ldots, j-1$. Define $\tau_{j}=\inf \left\{t \geq \tau_{j-1}: \delta_{j}(t) \leq e^{*}-\eta\right\}$. We show by a similar argument to that for $\tau_{1}$, that $\tau_{j}$ is finite. If $\tau_{j}=\tau_{j-1}$, then $\tau_{j}<\infty$ and $\delta_{j}\left(\tau_{j}\right) \leq e^{*}-\eta$. On the other hand, for regular points $t$ such that $\tau_{j-1} \leq t<\tau_{j}$ we have $e^{*}-\eta<\delta_{j}(t) \leq d_{1}^{*}(t) \leq e^{*}+\epsilon$. Note that we must have $j_{1}(t) \geq j$, since $t \geq \tau_{j-1}$ implies $\delta_{i}(t) \leq e^{*}-\eta$ for $i<j$. If $j_{1}(t)=j$, then by (4.9), $\dot{\delta}_{j}(t)=\psi_{j}\left(d_{1}^{*}(t)\right)-1 \leq \psi_{j}\left(e^{*}\right)-1<0$. On the other hand if $j<j_{1}(t)$, then from (4.28), $\dot{\delta}_{j}(t) \leq \psi_{j}\left(e^{*}\right)-1+\frac{\eta+\epsilon}{e^{*}+\epsilon}<0$. Thus, as for $\tau_{1}$, we have

$$
\tau_{j} \leq \tau_{j-1}+\frac{\epsilon+\eta}{1-\psi_{j}\left(e^{*}\right)-\frac{\eta+\epsilon}{e^{*}+\epsilon}}<+\infty .
$$

Finally, for any regular point $t \geq \tau_{j}$ for which $\delta_{j}(t) \in\left(e^{*}-\eta, e^{*}\right]$, by the same reasoning as above, we have $\dot{\delta}_{j}(t)<0$. It then follows that $\delta_{j}(t) \leq e^{*}-\eta$ for all $t \geq \tau_{j}$.

This completes the induction step and so the desired statement is true for all $j<j^{*}$, and so there is $\tau_{j^{*}-1}<\infty$ such that $\delta_{j}(t) \leq e^{*}-\eta$ for all $t \geq \tau_{j^{*}-1}$ and all $j<j^{*}$. Since we have assumed that $d_{1}^{*}(t)>e^{*}$ for all $t \geq 0$, it follows that for all $t \geq \tau_{j^{*}-1}$, we have $j_{1}(t) \geq j^{*}$. So if $j^{*}=N$, then $j_{1}(t)=j^{*}=N$ for all $t \geq \tau_{N-1}$, and we have proved the desired result.

We now suppose that $j^{*}<N$. We need to consider the behavior of the $\delta_{j}$ for $j>j^{*}$. We will show first that for all sufficiently large $t$, the first chokepoint is less than $N$, that is, $j_{1}(t)<N$. Let $t_{j^{*}-1}^{\prime}=\inf \left\{t \geq \tau_{j^{*}-1}: j_{1}(t)<N\right\}$. Note that $t_{j^{*}-1}^{\prime}$ is finite, by the same reasoning as that used to show that $j_{1}(t)=j^{*}$ for some finite time $t$. 
We now proceed by showing that for all sufficiently large $t$, the first chokepoint cannot be at $N$. We do this by showing that $\delta_{N}(t)<d_{1}^{*}(t)$ for all sufficiently large $t$. Note that since we have assumed $j^{*}<N$, and $C_{i}>0$ for all $i$, we have that $\alpha<e^{*}-\ell^{*}$. So for $\epsilon \in(0, \alpha)$, we have $\ell^{*}<e^{*}-\alpha+\epsilon<e^{*}$. Note also that whenever $j \neq j^{*}$, if $u \geq e^{*}-\eta>\ell^{*}$, then we have $\psi_{j}(u)-1 \leq \psi_{j}\left(e^{*}-\eta\right)-1<0$. Fix such an $\epsilon$, small enough that the prior induction proof also holds for this $\epsilon$. Let $\eta^{\prime}=\alpha-\epsilon$.

Now let $\tau_{1}^{\prime}=\inf \left\{t \geq t_{j^{*-1}}^{\prime}: \delta_{N}(t) \leq e^{*}-\eta^{\prime}\right\}$. We wish to show that $\tau_{1}^{\prime}$ is finite. If $\tau_{1}^{\prime}=t_{j^{*}-1}^{\prime}$ the results holds, so suppose that $t \in\left(t_{j^{*}-1}^{\prime}, \tau_{1}^{\prime}\right)$. Then $\delta_{N}(t)>e^{*}-\eta^{\prime}$. Since $\delta_{j_{1}(t)}(t)=d_{1}^{*}(t) \leq e^{*}+\epsilon$, we then immediately have $\delta_{j_{1}(t)}(t)-\delta_{N}(t) \leq \alpha$. Recall that $K(t)$ is the number of chokepoints at time $t$, with $N=j_{K(t)}$, so using (4.22), which also holds for $\left.d_{1}^{*}(0)>e^{*}\right)$, we have

$$
d_{1}^{*}(t)-d_{N}(t) \leq\left(\max _{j^{*} \leq n_{1}<n_{2} \leq N} \frac{C_{n_{2}}}{C_{n_{2}}-C_{n_{1}}}\right)\left(\delta_{j_{1}(t)}(t)-\delta_{N}(t)\right) \leq e^{*}-\ell^{*} .
$$

Hence, since $d_{1}^{*}(t)>e^{*}$, we immediately have $d_{N}(t)>\ell^{*}$. From (4.7) we have that at any regular point $t \in\left(t_{j^{*}-1}^{\prime}, \tau_{1}^{\prime}\right), \dot{\delta}_{N}(t)<\psi_{N}\left(d_{N}(t)\right)-1$, and now $d_{N}(t)>\ell^{*}$ implies further that $\psi_{N}\left(d_{N}(t)\right)-1<\psi_{N}\left(\ell^{*}\right)-1<0$. Then it follows that $\tau_{1}^{\prime}$ is finite, and furthermore, since, by similar reasoning to that above, $\dot{\delta}_{N}(t)<0$ for any regular point $t$ for which $\delta_{N}(t) \in\left(e^{*}-\eta^{\prime}, e^{*}\right]$, we have $\delta_{N}(t) \leq e^{*}-\eta^{\prime}$ for all $t \geq \tau_{1}^{\prime}$. Then, since we have assumed $d_{1}^{*}(t)>e^{*}$ for all $t \geq 0$, this implies that $j_{1}(t)<N$ for all $t \geq \tau_{1}^{\prime}$. Note that if $j^{*}=N-1$ then this completes the argument to show that $j_{1}(t)=j^{*}$ for all $t \geq \tau_{1}^{\prime}$. So we now assume that $j^{*}<N-1$.

In the above, we have shown that the first chokepoint is less than $N$ for all $t \geq \tau_{1}^{\prime}$. This implies that $j_{2}(t)$ exists for all times $t \geq \tau_{1}^{\prime}$.

Finally, we will prove the following concluding property: for $j=j^{*}+k, k=$ $1,2, \ldots, N-j^{*}$, there is a finite time (greater than or equal to $\tau_{1}^{\prime}$ ), after which $\delta_{j}(\cdot)$ is less than or equal to $e^{*}-\eta$ for all time. As for $j<j^{*}$, we shall use a proof by induction.

We will show first a general inequality, that will be used repeatedly in this section of the proof. We show that at any regular point $t \geq \tau_{1}^{\prime}$, for $j$ such that $j^{*}<j \leq j_{2}(t)$, if $\delta_{j}(t)>e^{*}-\eta$, then we have

$$
\dot{\delta}_{j}(t) \leq \psi_{j}\left(\ell^{*}\right)-1+\frac{\eta+2 \epsilon}{e^{*}+\epsilon}<0 .
$$

Note first that for any regular point $t \geq \tau_{1}^{\prime}$, if $j^{*}<j \leq j_{1}(t)$, and $\delta_{j}(t)>e^{*}-\eta$, then by (4.9) and (4.28), we have $\dot{\delta}_{j}(t) \leq \psi_{j}\left(e^{*}\right)-1+\frac{\eta+\epsilon}{e^{*}+\epsilon} \leq \psi_{j}\left(\ell^{*}\right)-1+\frac{\eta+2 \epsilon}{e^{*}+\epsilon}<0$, as desired. On the other hand, if $j^{*} \leq j_{1}(t)<j \leq j_{2}(t)$ then from (4.3),

$$
\delta_{j}(t)=\frac{C_{j_{1}(t)}}{C_{j}} \delta_{j_{1}(t)}(t)+\frac{M_{j}(t)-M_{j_{1}(t)}(t)}{C_{j}-C_{j_{1}(t)}} \frac{C_{j}-C_{j_{1}(t)}}{C_{j}} .
$$


Recalling that $e^{*}<d_{1}^{*}(t) \leq e^{*}+\epsilon$ and $d_{1}^{*}(t)=\delta_{j_{1}(t)}(t)$, if $\delta_{j}(t)>e^{*}-\eta$, then using (4.32) we have

$$
\begin{aligned}
\frac{M_{j}(t)-M_{j_{1}(t)}(t)}{C_{j}-C_{j_{1}(t)}} & >\left(e^{*}-\eta-\frac{C_{j_{1}(t)}(t)}{C_{j}} d_{1}^{*}(t)\right) \frac{C_{j}}{C_{j}-C_{j_{1}(t)}} \\
& =e^{*}-\left(d_{1}^{*}(t)-e^{*}\right) \frac{C_{j_{1}(t)}}{C_{j}-C_{j_{1}(t)}}-\eta \frac{C_{j}}{C_{j}-C_{j_{1}(t)}} \\
& \geq e^{*}-\frac{\epsilon C_{j_{1}(t)}+\eta C_{j}}{C_{j}-C_{j_{1}(t)}} \geq e^{*}-\frac{(\epsilon+\eta) C_{j}}{C_{j}-C_{j_{1}(t)}}
\end{aligned}
$$

So since $0<\epsilon<\eta<\frac{1}{2} \alpha$ and $e^{*}>\ell^{*}$, we have

$$
d_{2}^{*}(t) \geq \frac{M_{j}(t)-M_{j_{1}(t)}(t)}{C_{j}-C_{j_{1}(t)}}>\ell^{*}>\max _{j \neq j^{*}} e_{j} .
$$

For regular points $t \geq \tau_{1}^{\prime}$, if $j^{*}<j=j_{2}(t)$ and $\delta_{j}(t)>e^{*}-\eta$, then since $d_{2}^{*}(t)>$ $\ell^{*}>\max _{j \neq j^{*}} e_{j}$, from (4.7), $\dot{\delta}_{j}(t)<\psi_{j}\left(\ell^{*}\right)-1 \leq \psi_{j}\left(\ell^{*}\right)-1+\frac{\eta+\epsilon}{e^{*}+\epsilon}<0$. On the other hand, if $j^{*} \leq j_{1}(t)<j<j_{2}(t)$, and $\delta_{j}(t)>e^{*}-\eta$ at a regular point $t>\tau_{1}^{\prime}$, then

$$
\dot{\delta}_{j}(t)=\frac{1}{C_{j}} \sum_{i=1}^{j}\left(\rho_{i}\left(d_{i}(t)\right)-\Lambda_{i}(m(t))\right),
$$

where now

$$
\begin{aligned}
\frac{1}{C_{j}} \sum_{i=1}^{j} \Lambda_{i}(m(t)) & =\frac{1}{C_{j}}\left(C_{j_{1}(t)}+\sum_{i=j_{1}(t)+1}^{j} \frac{m_{i}(t)}{d_{2}^{*}(t)}\right) \\
& =\frac{C_{j_{1}(t)}}{C_{j}}+\frac{C_{j}-C_{j_{1}(t)}}{C_{j}} \frac{\frac{M_{j}(t)-M_{j_{1}(t)}(t)}{C_{j}-C_{j_{1}(t)}}}{d_{2}^{*}(t)} .
\end{aligned}
$$

We can use the bound $d_{2}^{*}(t)<d_{1}^{*}(t) \leq e^{*}+\epsilon$, together with (4.33) to obtain

$$
\frac{\frac{M_{j}(t)-M_{j_{1}(t)}(t)}{C_{j}-C_{j_{1}(t)}}}{d_{2}^{*}(t)}>\frac{e^{*}}{e^{*}+\epsilon}-\frac{\eta+\epsilon}{e^{*}+\epsilon} \frac{C_{j}}{C_{j}-C_{j_{1}(t)}} .
$$

We also have $\rho_{i}\left(d_{i}(t)\right) \leq \rho_{i}\left(d_{2}^{*}(t)\right)$ for all $i \leq j_{2}(t)$, since $d_{i}(t)=d_{2}^{*}(t)$ for $j_{1}(t)<$ $i \leq j_{2}(t)$ and $d_{1}(t)=\bar{d}_{1}^{*}(t)>d_{2}^{*}(t)$ for $i \leq j_{1}(t)$, and so from (4.35)-(4.38), and using the inequalities $\eta<\frac{1}{2} e^{*}(1-\theta)$ and $\epsilon<\left(e^{*}(1-\theta)-\eta\right) / 2$, 


$$
\begin{aligned}
\dot{\delta}_{j}(t) & \leq \psi_{j}\left(d_{2}^{*}(t)\right)-\left(\frac{C_{j_{1}(t)}}{C_{j}}+\left(\frac{C_{j}-C_{j_{1}(t)}}{C_{j}}\right)\left(\frac{e^{*}}{e^{*}+\epsilon}-\frac{\eta+\epsilon}{e^{*}+\epsilon} \frac{C_{j}}{C_{j}-C_{j_{1}(t)}}\right)\right) \\
& \leq \psi_{j}\left(\ell^{*}\right)-1+\left(1-\frac{C_{j_{1}(t)}}{C_{j}}\right)\left(1-\frac{e^{*}}{e^{*}+\epsilon}\right)+\frac{\eta+\epsilon}{e^{*}+\epsilon} \\
& \leq \psi_{j}\left(\ell^{*}\right)-1+\frac{\eta+2 \epsilon}{e^{*}+\epsilon} \leq \theta-1+\frac{\eta+2 \epsilon}{e^{*}+\epsilon}<0 .
\end{aligned}
$$

This proves the desired result (4.31) for $j^{*} \leq j_{1}(t)<j \leq j_{2}(t)$.

We now start the proof of the concluding property, which will proceed by induction. For the case $k=1$, we start by showing that $\tau_{j^{*}+1}=\inf \left\{t \geq \tau_{1}^{\prime}: \delta_{j^{*}+1}(t) \leq e^{*}-\eta\right\}$ is finite. If $\delta_{j^{*}+1}\left(\tau_{1}^{\prime}\right) \leq e^{*}-\eta$ then $\tau_{j^{*}+1}=\tau_{1}^{\prime}<\infty$. So suppose $\delta_{j^{*}+1}\left(\tau_{1}^{\prime}\right)>e^{*}-\eta$. Since we have already shown that for all $t \geq \tau_{1}^{\prime}, j_{1}(t) \geq j^{*}$, we have immediately that $j^{*}+1 \leq j_{2}(t)$ for all $t \geq \tau_{1}^{\prime}$. Hence for any regular points $t \geq \tau_{1}^{\prime}$, if $\delta_{j^{*}+1}(t)>e^{*}-\eta$, then by the property just proved, $\dot{\delta}_{j^{*}+1}(t) \leq \psi_{j^{*}+1}\left(\ell^{*}\right)-1+\frac{\eta+2 \epsilon}{e^{*}+\epsilon}<0$, and

$$
\tau_{j^{*}+1} \leq \tau_{1}^{\prime}+\frac{\eta+\epsilon}{1-\psi_{j^{*}+1}\left(\ell^{*}\right)-\frac{\eta+2 \epsilon}{e^{*}+\epsilon}}<\infty
$$

Furthermore, since $\dot{\delta}_{j^{*}+1}(t)<0$ for any regular point $t>\tau_{j^{*}+1}$ such that $\delta_{j^{*}+1}(t)>$ $e^{*}-\eta$, we have $\delta_{j^{*}+1}(t) \leq e^{*}-\eta$ for all $t \geq \tau_{j^{*}+1}$. Thus we have proved the case $k=1$ holds.

Now assume that the concluding property holds for $k \leq n-1$, some $n \geq 2$ and $n \leq N-j^{*}$. Then we may assume that for $j=j^{*}+1, j^{*}+2, \ldots, j^{*}+n-1$, $\delta_{j}(t) \leq e^{*}-\eta$ for all $t \geq \tau_{j^{*}+n-1}$. This implies, since $j_{1}(t) \geq j^{*}$, that if for some $t \geq \tau_{j^{*}+n-1}$ we have $\delta_{j^{*}+n}(t)>e^{*}-\eta$, then we will also have $j_{2}(t) \geq j^{*}+n$. Let $\tau_{j^{*}+n}=\inf \left\{t \geq \tau_{j^{*}+n-1}: \delta_{j^{*}+n}(t) \leq e^{*}-\eta\right\}$. If $\delta_{j^{*}+n}\left(\tau_{j^{*}+n-1}\right) \leq e^{*}-\eta$, then $\tau_{j^{*}+n}=\tau_{j^{*}+n-1}<\infty$. So we may suppose that $\delta_{j^{*}+n}\left(\tau_{j^{*}+n-1}\right)>e^{*}-\eta$. For any regular points $t \geq \tau_{j^{*}+n-1}$, if $\delta_{j^{*}+n}(t)>e^{*}-\eta$, then since that implies $j_{2}(t) \geq j^{*}+n$, by (4.31) we have $\dot{\delta}_{j^{*}+n}(t) \leq \psi_{j^{*}+n}\left(\ell^{*}\right)-1+\frac{\eta+2 \epsilon}{e^{*}+\epsilon}<0$, and

$$
\tau_{j^{*}+n} \leq \tau_{j^{*}+n-1}+\frac{\eta+\epsilon}{1-\psi_{j^{*}+n}\left(\ell^{*}\right)-\frac{\eta+2 \epsilon}{e^{*}+\epsilon}}<\infty .
$$

Furthermore, since $\dot{\delta}_{j^{*}+n}(t)<0$, for any regular point $t>\tau_{j^{*}+n}$ such that $\delta_{j^{*}+n}(t)>$ $e^{*}-\eta$, we have $\delta_{j^{*}+n}(t) \leq e^{*}-\eta$ for all $t \geq \tau_{j^{*}+n}$. Thus we have proved the case $k=n$ holds, and hence by the principle of induction, the concluding property holds for all $k=1,2, \ldots N-j^{*}$.

We have shown that for all $t \geq \tau_{N}$, we have $\delta_{j}(t) \leq e^{*}-\eta$ for all $j \neq j^{*}$. However, since $d_{1}^{*}(t) \geq e^{*}$, we must therefore have $\delta_{j^{*}}(t)=d_{1}^{*}(t)>e^{*}$ for all $t \geq \tau_{N}$.

Thus, finally, considering both the case $d_{1}^{*}(0)>e^{*}$ and $d_{1}^{*}(t) \leq e^{*}$ we have shown that $j_{1}(t) \rightarrow j^{*}$ as $t \rightarrow \infty$. Since $j_{1}(t)$ is integer valued, this implies that $j_{1}(t)=j^{*}$ for all $t$ sufficiently large. 


\section{Generalizations and Discussion}

\subsection{Living with Short Ramps}

In practice, onramps will be of different lengths, depending on their location. There are other reasons why some onramps may need to have shorter queues, for example, at peak times queues for an onramp may obstruct suburban streets.

A shorter ramp may act as a deterrent to arrivals. This can be incorporated in our model via the arrival rate function for that ramp. Furthermore a mechanism which allows our control policy to adjust the relative queue lengths and to achieve other effects is assigning weights to the queues. That is the topic of this subsection.

Let $w \in \mathbb{R}_{+}^{N}$ be weights attached to the queues. Then for fixed $m \in \mathbb{R}_{+}^{N}, \Lambda(m)$ is chosen to be a minimizer for the following adjusted minimization problem.

$$
\operatorname{minimize}_{\Lambda \in \mathbb{R}_{+}^{N}} \max _{1 \leq i \leq N}\left\{\frac{w_{i} m_{i}}{\Lambda_{i}}\right\} \text { such that } \sum_{i: i \leq j} \Lambda_{i} \leq C_{j} \text { for } 1 \leq j \leq N .
$$

The $w$-minmax delay controller $L P$ is: Given $m \in \mathbb{R}_{+}^{N}$,

$$
\begin{array}{r}
\tilde{d}_{1}^{*}(m)=\min _{\lambda \in \mathbb{R}_{+}^{N}} d \\
0 \leq w_{i} m_{i} \leq \lambda_{i}, \quad 1 \leq i \leq N, \\
0 \leq \sum_{i: i \leq j} \lambda_{i} \leq d C_{j}, \quad 1 \leq j \leq N .
\end{array}
$$

The solution to this has the same properties as did the unweighted controller: for queues $i \leq \tilde{j}_{1}(m)$, where $\tilde{j}_{1}(m)$ is the first chokepoint, we have that the delay, $\tau_{i}(m)$, at onramp $i$ satisfies

$$
w_{i} \tau_{i}(m)=\tilde{d}_{1}^{*}(m), \quad \text { for } 1 \leq i \leq j_{1}(m) .
$$

This is because the $w$-minmax and the minmax solutions map perfectly to each other via:

$$
\tilde{d}_{1}^{*}(m)=d_{1}^{*}(w m) \quad \text { and } \quad \tilde{j}_{1}^{*}(m)=j_{1}^{*}(w m) .
$$

\subsection{Control Policy when There are Outflows}

We have assumed throughout that all traffic admitted to the motorway will travel through to the end of the motorway. However, in general not all individuals will do so. In this section we outline an approach to incorporating outflow from the motorway, that is, we have offramps with nonzero traffic on them.

Suppose there is an outflow rate $y_{i} \geq 0$ for offramp $i$. In this section we write the onflow rate at onflow ramp $i$ to the motorway as $\Lambda_{i}(m, y)$. The effective onflow rate is 


$$
\hat{\Lambda}_{i}(m, y)=\Lambda_{i}(m, y)-y_{i}
$$

The delay is now

$$
d_{i}(m, y)=\frac{m_{i}}{\Lambda_{i}(m, y)}
$$

The congestion constraint becomes

$$
0 \leq \sum_{i=1}^{j} \hat{\Lambda}_{i}(m, y) \leq C_{j}
$$

which includes the requirement that the rate of flow of cars onto the motorway always be nonnegative. Additional constraints are

$$
0 \leq d_{i}(m, y) \leq d, 1 \leq i \leq N
$$

This now gives us the following minmax delay controller $L P$. Given $m, y \in \mathbb{R}_{+}^{N}$,

$$
\begin{gathered}
d_{1}^{*}(m, y)=\min _{\lambda \in \mathbb{R}_{+}^{N}} d \\
0 \leq m_{i} \leq \lambda_{i}, \quad 1 \leq i \leq N \\
0 \leq \sum_{i: i \leq j}\left(\lambda_{i}-d y_{i}\right) \leq d C_{j}, \quad 1 \leq j \leq N .
\end{gathered}
$$

When the optimal value $d_{1}^{*}(m, y) \neq 0$, for an optimal solution $\lambda(m, y)$, we define the control policy $\Lambda_{i}(m, y)$ for $i=1,2, \ldots, N$, by

$$
\Lambda_{i}(m, y):= \begin{cases}\frac{\lambda_{i}(m, y)}{d_{1}^{*}(m, y)} & \text { if } m_{i}>0 \\ 0 & \text { if } m_{i}=0\end{cases}
$$

(Note that an optimal solution may not be unique, see Sect. 2.2.2.)

\subsubsection{Properties and Analysis}

Thus the LP is the same as before except that the last constraint has been modified. This final constraint can be rewritten as

$$
d \sum_{i: i \leq j} y_{i} \leq \sum_{i: i \leq j} \lambda_{i} \leq d \hat{C}_{j}, \quad 1 \leq j \leq N .
$$

where $\hat{C}_{j}=C_{j}+\sum_{i: i \leq j} y_{i}$. The effect of the outflows is mostly to increase the freeway capacity.

Note The key hypothesis that capacity is increasing in the queue number still holds

$$
\hat{C}_{j+1}-\hat{C}_{j}=C_{j+1}-C_{j}+y_{j+1}>0 .
$$


Next we ask what parts of the theory that we developed in the previous sections still hold. Suppose the outflows $y_{i}$ are all constant in time. Then it is natural to define the equilibrium delays $\hat{e}_{1}, \ldots, \hat{e}_{N}$ by

$$
\sum_{i: i \leq j} \rho_{i}\left(\hat{e}_{j}\right)=C_{j}+\sum_{i: i \leq j} y_{i}=\hat{C}_{j}, \quad 1 \leq j \leq N .
$$

A plausible sufficient condition for the theorems to hold is that the lower bound in (5.12) is never an active constraint. Thus, for instance, we expect the convergence results of Theorem 1.3 will hold for suitable initial values $m(0)$, namely, those for which no resulting $m(t)$ and $j$ satisfy

$$
\sum_{i: i \leq j} y_{i}=\sum_{i: i \leq j} \Lambda_{i}(m(t), y)
$$

If this lower bound is achieved for some $j_{0}$, then the rate of flow of traffic onto the motorway is zero at $j_{0}$. Effectively, this would mean that congestion downstream of $j_{0}$ doesn't depend on traffic at $j_{0}$ or upstream of it. Thus it would be sufficient to consider the downstream network in isolation. In this paper we are particularly concerned with heavily congested networks, so it is reasonable to make the assumption that the lower bound is never the active constraint.

\subsection{Proportionally Fair}

The controller we studied here minimizes a worst case performance measure (maximum delay). Another possible way to choose the control policy $\Lambda$ is to maximize proportional fairness. Namely, for each $m$, a metering policy $\Lambda(m)$ is called proportionally fair (see [12]) if it solves

$$
\max _{\Lambda \in \mathbb{R}_{+}^{N}} \sum_{i=1}^{N} m_{i} \log \Lambda_{i}
$$

subject to

$$
\sum_{i: i \leq j} \Lambda_{i} \leq C_{j}, \quad 1 \leq j \leq N
$$

Here the $i^{\text {th }}$ summand in the objective function is assumed to be zero if $m_{i}=0$. This model with a proportionally fair policy has not been analyzed to date.

It would be interesting to compare the dynamic behavior of a proportionally fair controlled system with our minmax delay controlled system. 


\subsection{Discontinuity of $\Lambda_{i}$ at $m_{i}=0$ Downstream}

In another direction we point out that there are many natural values for a controller $\Lambda_{i}$ whenever $m_{i}=0$ at an $i$ which is downstream of the first choke point. This is because the limit of $\Lambda_{i}(m)$ as $m_{i} \rightarrow 0$ can take a range of values, and, hence $\Lambda_{i}$ need not be continuous in $m_{i}$ at 0 . (Remember that we have defined $\Lambda_{i}(m)=0$ when $m_{i}=0$.) We illustrate this with a couple of simple examples. The first example illustrates that continuity is possible. The next two illustrate how it can fail. Suppose $N=2$.

1. Suppose $m=(\epsilon, s)$, where $\epsilon<<s$. Then

$$
\Lambda_{1}(m)=C_{2} * \epsilon /(s+\epsilon) \quad \text { with } \quad \Lambda_{2}(m)=C_{2} * s /(s+\epsilon)
$$

and $\Lambda_{1}(m) \rightarrow 0$ as $\epsilon \rightarrow 0$.

2. Suppose $m=(s, \epsilon)$ where $\epsilon<<s$. Then $\Lambda_{1}(m)=C_{1}$ with $\Lambda_{2}(m)=C_{2}-C_{1}$ and $\Lambda_{2}(m) \rightarrow C_{2}-C_{1}$ as $\epsilon \rightarrow 0$.

3. Suppose $m=(a \epsilon, \epsilon)$ with $\epsilon$ small and for some $a \in \mathbb{R}_{+}$such that $a<C_{1} /\left(C_{2}-C_{1}\right)$ so that $j_{1}=2$. Then $\Lambda_{1}(m)=C_{2} * a /(1+a)$ and $\Lambda_{2}(m)=C_{2} /(1+a)$, neither of which converges to 0 as $\epsilon \rightarrow 0$.

Funding This research was supported in part by National Science Foundation Grants DMS-1500835, DMS1206772, DMS-1712974, the Charles Lee Powell Foundation, Matatini Marsden Fund grant UOA1114 and Te Pūnaha Matatini.

\section{Declarations}

Conflict of interest The authors declare that they have no competing interest.

Open Access This article is licensed under a Creative Commons Attribution 4.0 International License, which permits use, sharing, adaptation, distribution and reproduction in any medium or format, as long as you give appropriate credit to the original author(s) and the source, provide a link to the Creative Commons licence, and indicate if changes were made. The images or other third party material in this article are included in the article's Creative Commons licence, unless indicated otherwise in a credit line to the material. If material is not included in the article's Creative Commons licence and your intended use is not permitted by statutory regulation or exceeds the permitted use, you will need to obtain permission directly from the copyright holder. To view a copy of this licence, visit http://creativecommons.org/licenses/by/4.0/.

\section{References}

1. Abouaissa, H., Fliess, M., Join, C.: On ramp metering: towards a better understanding of ALINEA via model-free control. Int. J. Control 90, 1018-1026 (2017)

2. Como, G., Nilsson, G.: On the well-posedness of dynamical flow networks with feedback-controlled outflows. Preprint, arXiv:2001.06097 (2020)

3. Coogan, S., Arcak, M.: A compartmental model for traffic networks and its dynamical behavior. IEEE Trans. Autom. Control 60, 2698-2703 (2015)

4. Daganzo, C.F.: The cell transmission model: a dynamic representation of highway traffic consistent with the hydrodynamic theory. Transp. Res. B 28, 269-287 (1994)

5. Daganzo, C.F.: The cell transmission model, Part II: network traffic. Transp. Res. B 29, 79-93 (1995)

6. Dai, J.G., Weiss, G.: Stability and instability of fluid models for reentrant lines. Math. Oper. Res. 21, 115-134 (1996) 
7. Federer, H.: Geometric Measure Theory. Springer, Berlin (1969)

8. Gibbens, R.J., Saatci, Y.: Data, modelling and inference in road traffic networks. Philos. Trans. R. Soc. A366, 1907-1919 (2008)

9. Gomes, G., Horowitz, R., Kurzhanskiy, A.A., Varaiya, P., Kwon, J.: Behavior of the cell transmission model and effectiveness of ramp metering. Transp. Res. C 16, 485-513 (2008)

10. Huang, S., James, M.R.: $L^{\infty}$-bounded robustness for nonlinear systems: analysis and synthesis. IEEE Trans. Automatic Control 48, 1875-1891 (2003)

11. Huang, S., James, M.R., Jiang, Z.P.: L-infinity bounded robust control of nonlinear cascade systems. Syst. Control Lett. 54, 215-224 (2005)

12. Kelly, F.P., Williams, R.J.: Heavy traffic on a controlled motorway. In: Bingham, N.H., Goldie, C.M. (eds.) Probability and Mathematical Genetics: Papers in Honour of Sir John Kingman. Cambridge University Press, Cambridge (2010)

13. Levinson, D., Zhang, L.: Ramp meters on trial: evidence from the Twin Cities metering holiday. Transp. Res. A40, 810-828 (2006)

14. Mandjes, M., Storm, J.: A diffusion-based analysis of a multiclass road traffic network. Stoch. Syst. (2021). https://doi.org/10.1287/stsy.2019.0065

15. Papageorgiou, M., Kotsialis, A.: Freeway ramp metering: an overview. IEEE Trans. Intell. Transp. Syst. 3, 271-281 (2002)

16. Sumalee, A., Zhong, R.X., Pan, T.L., Szeto, W.Y.: Stochastic cell transmission model (SCTM): a stochastic dynamic traffic model for traffic state surveillance and assignment. Transp. Res. B 45, 507-533 (2011)

17. Varaiya, P.: What we've learned about highway congestion. Access 27, 2-9 (2005)

18. Zhang, L., Levinson, D.: Ramp metering and the capacity of active freeway bottlenecks. In: Proceedings of the 83rd Annual Meeting of the Transportation Research Board (2004)

19. Zhang, G., Wang, Y.: Optimizing coordinated ramp metering: a preemptive hierarchical control approach. Comput.-Aided Civil Infrastruct. Eng. 28, 22-37 (2013)

Publisher's Note Springer Nature remains neutral with regard to jurisdictional claims in published maps and institutional affiliations. 